\title{
The photosphere and chromosphere of the RS Canum Venaticorum star, II Pegasi
}

\section{A multi-wavelength campaign in August/September 1992}

P.B. Byrne ${ }^{1}$, H. Abdul Aziz ${ }^{6, \star}$, P.J. Amado ${ }^{1}$, M.J. Arevalo ${ }^{7}$, S. Avgoloupis ${ }^{4}$ J.G. Doyle ${ }^{1}$, M.T. Eibe ${ }^{1}$, K.H. Elliott ${ }^{5}$, R.D. Jeffries ${ }^{5, \star \star}$, A.C. Lanzafame ${ }^{1, \star \star \star}$, C. Lazaro ${ }^{7}$, H.M. Murphy ${ }^{1}$, J.E. Neff ${ }^{3}$, K.P. Panov ${ }^{8}$, L.M. Sarro ${ }^{1,2}$, J.H. Seiradakis ${ }^{4}$, and R.E. Spencer ${ }^{6}$

1 Armagh Observatory, Armagh BT61 9DG, N. Ireland

${ }^{2}$ LAEFF, Vilspa, Madrid, Spain

3 Department of Astronomy, Pennsylvania State University, 525 Davey Laboratory, University Park, PA 16802, U.S.A.

4 University of Thessaloniki, Department of Physics, Section of Astrophysics, Astronomy and Mechanics, GR-54006, Greece

5 School of Physics and Space Research, University of Birmingham, Birmingham B15 2TT, UK

${ }^{6}$ University of Manchester, Jodrell Bank, Macclesfield, SK11 9DL, UK

7 Instituto de Astrofisica de Canarias, via Lactea, E-38200 La Laguna, Tenerife, Canary Islands, Spain

8 National Astronomical Observatory, Bulgarian Academy of Sciences, 72 Trakya Blvd., Sofia 1754, Bulgaria

Received April 28; accepted June 11, 1997

\begin{abstract}
We describe multi-wavelength, simultaneous observations of the RS CVn star, II Pegasi, most of which were obtained during the first three weeks of September 1992. These observations were made using optical and infra-red broad-band photometry, ultraviolet and optical spectroscopy and microwave monitoring. We have detected photospheric spots and chromospheric flares, as well as deriving a description of mean conditions in the quiet chromosphere. One of the flares, observed in optical photometry and ultraviolet spectroscopy is one of the most energetic ever observed on this star. We demonstrate that in its "quiescent" state II Peg is continually variable in most of its chromospheric emissions, as well as in its coronal output.
\end{abstract}

Key words: stars: late-type; activity; chromosphere; II Peg — radio continuum: stars

Send offprint requests to: P.B. Byrne

* Present address: School of Physics, Universiti Sains Malaysia, 11800 Penang, Malaysia.

** Present address: Dept. of Physics, Keele University, Staffordshire ST5 5BG, UK.

${ }^{\star \star \star}$ Present address: Instituto di Astronomia, Università di Catania, Viale A. Doria 6, I-95125 Catania, Italy.

\section{Introduction}

Chromospheric and coronal heating on the Sun is highly concentrated into localised active regions of enhanced magnetic field. The distribution of such active regions with solar longitude is highly non-uniform, with the global brightness in high-temperature spectral features sometimes being dominated by a few very active regions. This is especially true near the maximum of the Sun's magnetic cycle.

Chromospherically active late-type stars exhibit most of the characteristics of the active Sun but on a globally much enhanced scale. RSCVn stars are close latetype binaries in which one component lies above the main sequence and, forced into corotation through tidal interaction, is chromospherically active as a result of dynamo generation of magnetic fields. RS CVn's exhibit a wide range of solar-like activity phenomena. These include non-radiatively heated chromospheres and X-ray emitting coronae (Doyle et al. 1991, 1992a,b), cool surface spots (Byrne 1992a,b) and frequent flares (Doyle et al. 1989b).

Based on the solar experience, it might be expected that non-uniform distributions of magnetic heating on RS CVn stars would lead to variability in the stars' detected flux in suitable chromospheric and coronal radiations as the star rotates, i.e. rotational modulation. Such effects have been very elusive, however, in spite of much observational effort (Rodonó et al. 1987; Andrews et al. 1988; Byrne et al. 1987, 1989, 1995 (hereafter Paper I), Doyle et al. 1989a, 1992a,b). However, since most 
Table 1. Table of standard magnitudes and colours of II Peg derived from observations taken at Observatorio del Roque de los Muchachos in September 1992, at the Stephanion Observatory in both July and September 1992 and at the Bulgarian National Observatory in August 1992. Phases have been calculated according to the ephemeris of Vogt (1981), i.e. $\mathrm{JD}=2443033.47+6.72422 E$

\begin{tabular}{|c|c|c|c|c|c|c|c|c|}
\hline $\begin{array}{l}\text { Date } \\
1992\end{array}$ & $\overline{\mathrm{UT}}$ & $\begin{array}{c}\text { JD } \\
2440000.0+\end{array}$ & Phase & $\bar{V}$ & $\overline{B-V}$ & $\overline{U-B}$ & $V-R$ & $V-I$ \\
\hline \multicolumn{9}{|c|}{ Jacobus Kapteyn Telescope } \\
\hline 08 Sep & $23: 52$ & 8874.4945 & 0.655 & 7.522 & 1.022 & 0.798 & 0.599 & 1.220 \\
\hline 09 Sep & $05: 28$ & 8874.7277 & 0.689 & 7.500 & 1.022 & 0.877 & 0.645 & 1.275 \\
\hline \multirow[t]{2}{*}{$10 \mathrm{Sep}$} & $02: 13$ & 8875.5923 & 0.818 & 7.510 & 1.017 & 0.628 & 0.603 & 1.268 \\
\hline & $23: 58$ & 8876.4985 & 0.953 & 7.599 & 1.058 & 0.707 & 0.613 & 1.266 \\
\hline 12 Sep & $00: 15$ & 8877.5104 & 0.103 & 7.597 & 1.012 & 0.704 & 0.561 & 1.269 \\
\hline 13 Sep & $02: 18$ & 8878.5957 & 0.265 & 7.456 & 1.042 & 0.650 & 0.594 & 1.206 \\
\hline 14 Sep & 00:02 & 8879.5017 & 0.400 & 7.437 & 1.029 & 0.698 & 0.563 & 1.203 \\
\hline 15 Sep & 00:14 & 8880.5095 & 0.549 & 7.457 & 1.055 & 0.669 & 0.577 & 1.235 \\
\hline 16 Sep & $00: 50$ & 8881.5345 & 0.702 & 7.444 & 0.980 & 0.793 & 0.587 & 1.235 \\
\hline 17 Sep & 05:11 & 8882.7158 & 0.877 & 7.624 & 1.105 & 0.828 & 0.632 & 1.278 \\
\hline \multicolumn{9}{|c|}{ Stephanion Observatory } \\
\hline $25 \mathrm{Jul}$ & $00: 37$ & 8828.526 & 0.818 & 7.551 & 1.050 & 0.727 & & \\
\hline $27 \mathrm{Jul}$ & $00: 34$ & 8830.527 & 0.116 & 7.571 & 1.045 & 0.731 & & \\
\hline $28 \mathrm{Jul}$ & $00: 39$ & 8831.527 & 0.265 & 7.484 & 1.021 & 0.740 & & \\
\hline $29 \mathrm{Jul}$ & 00:41 & 8832.528 & 0.413 & 7.494 & 1.035 & 0.760 & & \\
\hline $30 \mathrm{Jul}$ & 00:39 & 8833.527 & 0.562 & 7.500 & 1.033 & 0.717 & & \\
\hline $31 \mathrm{Jul}$ & 00:42 & 8834.529 & 0.711 & 7.478 & 1.037 & 0.621 & & \\
\hline $31 \mathrm{Jul}$ & $23: 42$ & 8835.488 & 0.854 & 7.563 & 1.047 & 0.706 & & \\
\hline 03 Sep & $21: 58$ & 8869.415 & 0.899 & 7.552 & 1.052 & 0.710 & & \\
\hline 04 Sep & $22: 26$ & 8870.435 & 0.051 & 7.646 & 1.058 & 0.739 & & \\
\hline 05 Sep & 21:05 & 8871.378 & 0.191 & 7.496 & 1.008 & 0.468 & & \\
\hline 09 Sep & 01:46 & 8874.574 & 0.666 & 7.462 & 1.016 & 0.652 & & \\
\hline 11 Sep & $01: 29$ & 8876.562 & 0.962 & 7.608 & 1.049 & 0.717 & & \\
\hline 18 Sep & $22: 05$ & 8884.420 & 0.131 & 7.630 & 1.047 & 0.726 & & \\
\hline \multicolumn{9}{|c|}{ Bulgarian National Observatory } \\
\hline $26 \mathrm{Aug}$ & $01: 23$ & 8860.558 & 0.582 & 7.522 & 1.031 & 0.671 & & \\
\hline 27 Aug & $00: 56$ & 8861.539 & 0.728 & 7.497 & 1.030 & 0.653 & & \\
\hline 28 Aug & $00: 32$ & 8862.522 & 0.874 & 7.560 & 1.027 & 0.656 & & \\
\hline 29 Aug & 00:04 & 8863.503 & 0.020 & 7.652 & 1.057 & 0.704 & & \\
\hline 30 Aug & 00:42 & 8864.529 & 0.173 & 7.571 & 1.043 & 0.692 & & \\
\hline $30 \mathrm{Aug}$ & $22: 15$ & 8865.427 & 0.306 & 7.518 & 1.036 & 0.694 & & \\
\hline
\end{tabular}

previous efforts have been based on either sampling a single rotation of the active star, or random sampling during many different rotations, there is an obvious danger of any rotational modulation being masked by short-term variability, such as flaring, or longer-term variations, such as the growth and decay of active regions.

In this paper we describe observations of the $6.72 \mathrm{~d}$ SB1 RS CVn K2IV binary, II Peg in the ultraviolet, optical and microwave spectral regimes, over varying fractions of 2 stellar rotations, which are then used to examine these issues. In this paper we present the data resulting from these observations. In a forthcoming paper we will discuss their implications more fully (Byrne et al. in preparation). Note that throughout this paper we use the orbital ephemeris of Vogt (1981), i.e. JD = 2443033.47+6.72422E, which we found in Paper I to be more accurate than any of the other published ephemerides. We also assume, as have others, that II Peg's axial rotation is tidally locked to the orbital motion of its companion.

\section{Observations}

An extensive campaign of monitoring II Peg was organised in the third quarter of 1992. The core of the coordinated campaign took place during the two-week interval 5-19 September, but photometric observations were also made in both July and August to establish the phase and amplitude of the spot modulation prior to the main phase of the campaign.

\subsection{Photometry}

Visible and infra-red photometry was carried out at a number of observing sites between July and September 
1992. Standard photometry in Johnson $U B V$ (Johnson 1966), Cousins $R I_{\mathrm{C}}$ (Cousins 1976) and infra-red $J H K$ (Johnson 1966) as well as continuous monitoring for flares in the Johnson $U$ band were achieved.

\subsubsection{Photometry - Jacobus Kapteyn Telescope (JKT)}

We observed II Peg at the $1 \mathrm{~m}$ JKT at the Observatorio del Roque de los Muchachos on the island of La Palma in the Canary Islands, 7-16 September 1992. The telescope was equipped with an EEV $1280 \times 1180$ pxl CCD detector and glass filters, which together approximated to the Cape $U B V(R I)_{\mathrm{C}}$ photometric system. Equatorial standard stars from the lists of Menzies et al. (1991) were measured on each night to relate the instrumental to the standard system. The resulting magnitudes and colours are to be found in Table 1 .

II Peg, along with two nearby comparison stars, viz. SAO $91568\left(=\mathrm{BD}+28^{\circ} 4665\right)$ and $\mathrm{SAO} 91577$ $\left(=\mathrm{HD} 224085=\mathrm{BD}+28^{\circ} 4667\right)$, were measured in all five colours several times per night. These acted as comparison and check star, respectively. Their mean magnitudes and colours were found to be

SAO 91568: $V=8.507 ;(B-V)=0.751 ;(U-B)=0.474$
$(V-R)_{\mathrm{C}}=0.372 ;(V-I)_{\mathrm{C}}=0.752$

$$
(V-R)_{\mathrm{C}}=0.372 ;(V-I)_{\mathrm{C}}=0.752
$$

SAO 91577: $V=8.243 ;(B-V)=1.267 ;(U-B)=1.420$

$$
(V-R)_{\mathrm{C}}=0.614 ;(V-I)_{\mathrm{C}}=1.243 .
$$

The values for SAO 91577 may be compared with those determined by the present authors over several seasons (Rodonó et al. 1986; Andrews et al. 1988; Byrne et al. 1989; Doyle et al. 1989a, 1992a), viz.

$$
\begin{gathered}
\text { SAO 91577: } V=8.23 ;(B-V)=1.29 ;(U-B)=1.41 \\
(V-R)_{\mathrm{C}}=0.65 ;(V-I)_{\mathrm{C}}=1.24
\end{gathered}
$$

and with those found from our other photometry below.

Continuous monitoring of II Peg in the Johnson $U$ band was also undertaken at the JKT on 8 nights between 6/7-15/16 September 1992 (Byrne et al. 1994). The telescope was moved between individual exposures to create up to ten images of the star before reading out the CCD, thus reducing the overhead due to CCD readout. Details of the coverage achieved, which totalled $31.7 \mathrm{hr}$, will be found in Table 2. No flares were recorded in this time.

\subsubsection{Photometry - Stephanion Observatory}

Standard $U B V$ photometry and $U$-band flare monitoring were carried out at the Stephanion Observatory (SO), Greece, using the $75 \mathrm{~cm}$ Cassegrain reflector belonging to the University of Thessaloniki between July 24-31 and September 3-18, 1992. The telescope and photometer have been described by Mavridis et al. (1982). Nightly measurements were referred to the same two local comparison stars, i.e. SAO 91568 and SAO 91577, which in turn were referred to standard stars on the best 3 nights photometrically. Typical errors in $V$ and $B-V$ were $\sim 0.01$, while
Table 2. Flare monitoring times of II Peg at the Jacobus Kapteyn Telescope, 6-16 September 1992, and at Stephanion

\begin{tabular}{|c|c|c|c|}
\hline $\begin{array}{l}\text { Date } \\
1992\end{array}$ & \multicolumn{2}{|c|}{$\begin{array}{c}\text { Monitoring Intervals } \\
\text { UT }\end{array}$} & Total \\
\hline \multicolumn{4}{|c|}{ Jacobus Kapteyn Telescope } \\
\hline 6/7 Sep. & $04: 49-06: 07$ & & $01^{\mathrm{h}} 18^{\mathrm{m}}$ \\
\hline 7/8 Sep. & $03: 53-05: 17$ & & $01^{\mathrm{h}} 24^{\mathrm{m}}$ \\
\hline 8/9 Sep. & $\begin{array}{c}\mathbf{2 0 : 3 3}-\mathbf{2 0 : 3 6} \\
03: 57-05: 02\end{array}$ & $20: 42-23: 26$ & $03^{\mathrm{h}} 52^{\mathrm{m}}$ \\
\hline 10/11 Sep. & $20: 55-23: 11$ & & $02^{\mathrm{h}} 16^{\mathrm{m}}$ \\
\hline 12/13 Sep. & $02: 52-06: 01$ & & $03^{\mathrm{h}} 09^{\mathrm{m}}$ \\
\hline 13/14 Sep. & $20: 25-23: 46$ & $01: 32-06: 10$ & $07^{\mathrm{h}} 59^{\mathrm{m}}$ \\
\hline 14/15 Sep. & $21: 01-23: 20$ & $01: 58-06: 08$ & $06^{\mathrm{h}} 29^{\mathrm{m}}$ \\
\hline 15/16 Sep. & $21: 01-23: 34$ & $03: 00-05: 59$ & $05^{\mathrm{h}} 32^{\mathrm{m}}$ \\
\hline \multicolumn{4}{|c|}{ Stephanion Observatory } \\
\hline 4/5 Sep. & $\begin{array}{l}22: 24-23: 55 \\
01: 52-02: 35\end{array}$ & $00: 11-01: 36$ & $03^{\mathrm{h}} 08^{\mathrm{m}}$ \\
\hline 5/6 Sep. & $\begin{array}{l}19: 41-20: 42 \\
00: 00-01: 04\end{array}$ & $\begin{array}{l}21: 27-2343 \\
01: 10-01: 46\end{array}$ & $04^{\mathrm{h}} 09^{\mathrm{m}}$ \\
\hline 6/7 Sep. & $\begin{array}{l}19: 49-21: 19 \\
22: 16-01: 18\end{array}$ & $\begin{array}{l}2123-22: 06 \\
01: 29-01: 38\end{array}$ & $05^{\mathrm{h}} 10^{\mathrm{m}}$ \\
\hline 7/8 Sep. & $\begin{array}{l}20: 03-23: 36 \\
01: 56-02: 22\end{array}$ & $23: 40-01: 48$ & $05^{\mathrm{h}} 50^{\mathrm{m}}$ \\
\hline 8/9 Sep. & $\begin{array}{c}\text { 19:53-22:19 } \\
23: 23-23: 55 \\
00: 18-01: 31\end{array}$ & $\begin{array}{c}\mathbf{2 2 : 2 7}-\mathbf{2 3 : 1 4} \\
23: 59-00: 14\end{array}$ & $05^{\mathrm{h}} 03^{\mathrm{m}}$ \\
\hline 9/10 Sep. & $\begin{array}{l}19: 38-21: 55 \\
22: 40-22: 50\end{array}$ & $22: 01-22: 36$ & $02^{\mathrm{h}} 59^{\mathrm{m}}$ \\
\hline 10/11 Sep. & 20:16-01:11 & & $04^{\mathrm{h}} 40^{\mathrm{m}}$ \\
\hline 11/12 Sep. & $\begin{array}{l}19: 48-22: 40 \\
01: 22-02: 17\end{array}$ & $22: 46-01: 14$ & $04^{\mathrm{h}} 59^{\mathrm{m}}$ \\
\hline 12/13 Sep. & 19:09-19:57 & $21: 23-02: 26$ & $05^{\mathrm{h}} 29^{\mathrm{m}}$ \\
\hline 13/14 Sep. & $\begin{array}{c}18: 52-19: 46 \\
\mathbf{2 0 : 1 7 - 2 2 : 2 8}\end{array}$ & $\begin{array}{c}\text { 19:50-20:07 } \\
\mathbf{2 2 : 3 3 - 0 0 : 3 3}\end{array}$ & \\
\hline & $00: 37-01: 14$ & $01: 23-02: 23$ & $06^{\mathrm{h}} 44^{\mathrm{m}}$ \\
\hline 14/15 Sep. & $\begin{array}{l}20: 47-00: 29 \\
01: 34-02: 31\end{array}$ & $00: 39-01: 21$ & $05^{\mathrm{h}} 01^{\mathrm{m}}$ \\
\hline 15/16 Sep. & $\begin{array}{c}18: 49-20: 04 \\
\mathbf{2 2 : 4 3}-\mathbf{0 2 : 1 9}\end{array}$ & $20: 25-22: 38$ & $06^{\mathrm{h}} 43^{\mathrm{m}}$ \\
\hline 16/17 Sep. & $\begin{array}{l}18: 48-20: 00 \\
22: 09-23: 06 \\
01: 29-02: 20\end{array}$ & $\begin{array}{l}21: 14-22: 05 \\
23: 16-01: 10\end{array}$ & $05^{\mathrm{h}} 19^{\mathrm{m}}$ \\
\hline 17/18 Sep. & $\begin{array}{l}19: 03-19: 51 \\
01: 51-02: 18\end{array}$ & $20: 01-01: 33$ & $06^{\mathrm{h}} 32^{\mathrm{m}}$ \\
\hline 18/19 Sep. & $20: 15-21: 41$ & $22: 27-01: 46$ & $04^{\mathrm{h}} 25^{\mathrm{m}}$ \\
\hline
\end{tabular}
Observatory, 4-19 September 1992. Breaks of duration $\leq 3 \mathrm{~min}$ have been ignored. Times in bold are those during which both telescopes were observing for at least some of the time

those for $U-B$ were $\sim 0.04$. The following mean magnitudes and colours for these local comparisons were derived.

SAO 91568: $\quad V=8.525 ;(B-V)=0.781 ;(U-B)=0.453$ SAO 91577: $V=8.246 ;(B-V)=1.253 ;(U-B)=$ 1.239 .

These may be compared with our magnitudes for these same stars as measured at the JKT above (see Sect. 2.1.1 and Sect. 3.1.1). The resulting magnitudes and colours derived for II Peg will be found in Table 1.

Continuous monitoring of II Peg in the Johnson $U$ band was also undertaken at Stephanion on 15 nights 
between 4-19 September 1992. Details of the coverage achieved, which totalled $76.2 \mathrm{hr}$, will be found in Table 2 .

\subsubsection{Photometry - Bulgarian National Observatory}

Standard $U B V$ photometry was also carried out at the Bulgarian National Observatory's (BNO) $60 \mathrm{~cm}$ telescope at Rozhen between 25-30 August 1992. These observations were made differentially with respect to the local comparison star, SAO 91568, for which the standard magnitudes and colours measured previously were also assumed here as follows.

SAO 91568: $V=8.54 ;(B-V)=0.79 ;(U-B)=0.49$.

The resulting magnitudes and colours derived for II Peg will be found in Table 1 .

Table 3. Table of nightly mean standard $J, H$ and $K$ magnitudes of II Peg derived from the observations taken at the Observatorio del Teide, Tenerife, in July and August 1992. Phases have been calculated according to the ephemeris of Vogt $(1981)$, i.e. $\mathrm{JD}=2443033.47+6.72422 E$

\begin{tabular}{lcccccc}
\hline $\begin{array}{l}\text { Date } \\
1992\end{array}$ & UT & $\begin{array}{c}\text { JD } \\
2440000.0+\end{array}$ & Phase & $J$ & $H$ & $K$ \\
\hline 31 Jul. & $04: 20$ & 8834.681 & 0.734 & 5.412 & 4.802 & 4.628 \\
1 Aug. & $03: 23$ & 8835.641 & 0.876 & 5.411 & 4.802 & 4.623 \\
2 Aug. & $03: 39$ & 8836.652 & 0.027 & 5.455 & 4.842 & 4.662 \\
3 Aug. & $02: 54$ & 8837.621 & 0.171 & 5.419 & 4.803 & 4.627 \\
5 Aug. & $01: 26$ & 8839.560 & 0.459 & 5.407 & 4.788 & 4.599 \\
6 Aug. & $03: 00$ & 8840.625 & 0.618 & 5.392 & 4.775 & 4.597 \\
7 Aug. & $02: 42$ & 8841.612 & 0.765 & 5.400 & 4.782 & 4.604 \\
8 Aug. & $00: 41$ & 8842.528 & 0.901 & 5.452 & 4.821 & 4.650 \\
9 Aug. & $00: 48$ & 8843.533 & 0.050 & 5.441 & 4.811 & 4.642 \\
10 Aug. & $03: 21$ & 8844.640 & 0.215 & 5.389 & 4.786 & 4.620 \\
11 Aug. & $02: 35$ & 8846.607 & 0.507 & 5.395 & 4.780 & 4.605 \\
12 Aug. & $03: 51$ & 8847.660 & 0.664 & 5.389 & 4.766 & 4.599 \\
15 Aug. & $04: 40$ & 8850.694 & 0.115 & 5.441 & 4.825 & 4.649 \\
\hline
\end{tabular}

\subsubsection{Photometry - Infra-red}

Infra-red photometry of II Peg was carried out using the $1.5 \mathrm{~m}$ Carlos Sanchez telescope at the Observatorio del Teide of the Instituto de Astrofisica de Canarias (IAC) at Mt. Teide, Tenerife (Canary Islands) on 13 nights between 30 July and 14 August 1992. A CVF photometer with a focal plane chopper was used. The detector was a liquid-nitrogen-cooled InSb device which, together with filters, gave a good approximation to the standard $J, H$ and $K$ bands (Johnson 1966; Glass 1985). Both the chopping amplitude and the aperture diameter were 15 arcsec. Each photometric measurement was performed exposing the star for $10 \mathrm{~s}$ in alternate beams until the signal-tonoise of the integrated measurement was $\geq 500$. The comparison star was SAO 91577. Its standard magnitudes were found to be
SAO 91577: $J=6.15 ; \quad H=5.84 ; K=5.32 ; \quad L=5.17$.

Standard errors on each measurement were $\sim 0.01$.

Standard reference stars were also observed to calibrate the observations to the standard system. A log of this data will be found in Table 3 .

\subsection{Optical spectroscopy}

Three optical spectroscopic data sets were taken during the campaign. The first consisted of high-resolution spectra, one per night, of Balmer $\mathrm{H} \alpha$ and $\mathrm{H} \beta$, as well as $\mathrm{HeI} \mathrm{D}_{3}$. The second was in the blue at lower resolution and included the higher members of the Balmer series and the CaII H\&K doublet. The third comprised a time sequence of $\mathrm{H} \alpha$ spectra, also at a lower resolution.

\subsubsection{Optical spectroscopy - high-resolution data}

High-resolution $(R \sim 25000)$ spectroscopy was carried out using the Solar Stellar Spectrograph at the US National Solar Observatory's 1.6m McMath Pierce Telescope at Kitt Peak, Arizona on 5 nights between 11-16 September 1992. On all clear nights a single spectrum was recorded in the region of the Balmer $\mathrm{H} \alpha$ line, while on a smaller number of nights spectra were recorded near Balmer $\mathrm{H} \beta$ and $\mathrm{HeID}_{3}$. A log of these spectra will be found in Table 4 .

Table 4. A log of the optical high-resolution spectra recorded at the McMath Pierce Telescope between 11-16 September and of blue low-resolution spectra at the Isaac Newton Telescope between 14-19 September 1992. Phases have been calculated according to the ephemeris of Vogt (1981)

\begin{tabular}{lccccc}
\hline $\begin{array}{c}\text { Date } \\
1992\end{array}$ & UT & Exp & JD & Phase & $\lambda_{\mathrm{c}}$ \\
mid-exp & min & 2440000.0+ & & $\AA$ \\
\hline \multicolumn{6}{c}{ McMath Pierce Telescope } \\
\hline 11 Sep. & $04: 57$ & 30 & 8876.706 & 0.983 & 6562.8 \\
& $06: 55$ & 30 & 8876.788 & 0.996 & 5975.7 \\
& $08: 52$ & 30 & 8876.869 & 0.008 & 4861.3 \\
12 Sep. & $08: 47$ & 40 & 8877.866 & 0.156 & 6562.8 \\
& $09: 57$ & 30 & 8877.915 & 0.163 & 4861.3 \\
& $11: 39$ & 35 & 8877.985 & 0.174 & 5975.7 \\
14 Sep. & $08: 47$ & 30 & 8879.866 & 0.453 & 6562.8 \\
& $10: 04$ & 35 & 8879.919 & 0.461 & 5975.7 \\
15 Sep. & $08: 35$ & 40 & 8880.858 & 0.601 & 6562.8 \\
16 Sep. & $08: 28$ & 07 & 8881.853 & 0.749 & 6562.8 \\
\hline \multicolumn{6}{c}{ Isaac Newton Telescope } \\
\hline 15 Sep. & $02: 00$ & 20.0 & 8880.583 & 0.560 & 4128 \\
16 Sep. & $01: 56$ & 16.7 & 8881.581 & 0.708 & 4128 \\
17 Sep. & $02: 00$ & 16.7 & 8882.583 & 0.858 & 4128 \\
18 Sep. & $02: 01$ & 16.7 & 8883.584 & 0.006 & 4128 \\
20 Sep. & $01: 56$ & 16.7 & 8885.581 & 0.303 & 4128 \\
\hline
\end{tabular}


Table 5. A $\log$ of the optical low-resolution $\mathrm{H} \alpha$ spectra recorded at the University of Birmingham's Wast Hills Observatory between 7-20 September 1996. Phases have been calculated according to the ephemeris of Vogt (1981)

\begin{tabular}{rccc}
\hline $\begin{array}{r}\text { Date } \\
1992\end{array}$ & UT & JD & Phase \\
\hline 7/8 Sep. & $21: 53-04: 35$ & $8873.412-.691$ & $0.494-0.535$ \\
8/9 Sep. & $20: 47-22: 31$ & $8874.366-.483$ & $0.635-0.653$ \\
10/11 Sep. & $21: 39-01: 32$ & $8876.402-.564$ & $0.938-0.962$ \\
11/12 Sep. & $20: 38-01: 12$ & $8877.360-.550$ & $0.081-0.109$ \\
13/14 Sep. & $20: 16-04: 16$ & $8879.343-.678$ & $0.376-0.425$ \\
14/15 Sep. & $20: 04-02: 35$ & $8880.336-.608$ & $0.523-0.564$ \\
15/16 Sep. & $23: 54-04: 49$ & $8881.496-.701$ & $0.696-0.726$ \\
16/17 Sep. & $20: 09-21: 22$ & $8882.340-.390$ & $0.821-0.829$ \\
20/21 Sep. & $20: 30-21: 26$ & $8886.353-.393$ & $0.418-0.424$ \\
\hline
\end{tabular}

\subsubsection{Optical spectroscopy - blue low-resolution data}

Low-resolution $(R \sim 4500)$ spectroscopic data in the blue region $(\lambda 3890-4365 \AA)$ were obtained using the Intermediate Dispersion Spectrograph (IDS) on the $2.5 \mathrm{~m}$ Isaac Newton Telescope (INT) at the Observatorio del Roque de los Muchachos on the island of La Palma on five nights between 1992 September 14-19. A 2400 lines/mm grating, blazed at $3500 \AA$ was used and a slit width of 1.35 arcsec, which projected to $2.2 \mathrm{pxl}$ on the CCD detector. A log of these spectra will be found in Table 4 .

\subsubsection{Optical spectroscopy - red low-resolution data}

Low-resolution $(R \sim 3300) \mathrm{H} \alpha$ data were recorded on 9 nights between 7-20 September 1992 using the $40 \mathrm{~cm}$ telescope at the University of Birmingham's Wast Hills Observatory. The telescope was equipped with a zoom lens spectrograph (Elliott 1996) equipped with a GEC CCD detector which gave 385 pixels in the dispersion direction, corresponding to a wavelength coverage of $\sim 760 \AA$. A log of this data will be found in Table 5 .

\section{3. $U V$ spectroscopy}

Ultraviolet spectroscopy was obtained with the International Ultraviolet Explorer satellite (IUE) (Boggess et al. 1978) on 12 consecutive days between 1992 September 5-16. Data were obtained using both the IUE's long-wavelength $(\approx 1900-3200 \AA$; LWP $)$ and shortwavelength $(\approx 1150-1950 \AA$; SWP $)$ cameras in high$(R \approx 45000$ at $\lambda 2800 \AA$; HIRES $)$ and low-resolution $(R \approx 300$ at $\lambda 1500 \AA$; LORES), respectively. Between three and six spectra were obtained on each date alternating between LWP HIRES and SWP LORES. This observing scheme enabled us to make maximum use of the satellite as SWP exposures were made while the LWP camera was being read to ground and prepared for the next obser-
Table 6. Log of the IUE spectra of II Peg obtained between 1992 September 5-16. Phases have been calculated according to the ephemeris of Vogt (1981)

\begin{tabular}{|c|c|c|c|c|}
\hline $\begin{array}{l}\text { Date } \\
1992\end{array}$ & $\begin{array}{c}\text { IUE Image } \\
\text { No. }\end{array}$ & $\begin{array}{c}\text { UT } \\
\text { Start }\end{array}$ & $\begin{array}{l}\text { Exp. } \\
\text { min. }\end{array}$ & $\begin{array}{c}\text { JD (mid-exp) } \\
2440000.0+\end{array}$ \\
\hline \multirow{3}{*}{5 Sep. } & SWP45531 & $19: 40: 33$ & 20 & 8871.327 \\
\hline & LWP23854 & 20:19:41 & 30 & 8871.357 \\
\hline & SWP45532 & $20: 58: 30$ & 107 & 8871.411 \\
\hline \multirow[t]{3}{*}{6 Sep. } & LWP23864 & $19: 37: 39$ & 30 & 8872.328 \\
\hline & SWP45543 & $20: 17: 32$ & 100 & 8872.380 \\
\hline & LWP23865 & $22: 06: 18$ & 30 & 8872.431 \\
\hline \multirow[t]{4}{*}{7 Sep. } & LWP23873 & 19:20:32 & 30 & 8873.316 \\
\hline & SWP45553 & 19:59:17 & 100 & 8873.418 \\
\hline & LWP23874 & $21: 47: 16$ & 30 & 8873.368 \\
\hline & SWP45554 & $22: 22: 56$ & 25 & 8873.441 \\
\hline \multirow[t]{6}{*}{8 Sep. } & LWP23875 & $15: 51: 00$ & 30 & 8874.171 \\
\hline & SWP45571 & $16: 28: 51$ & 100 & 8874.221 \\
\hline & LWP23876 & $18: 23: 12$ & 30 & 8874.277 \\
\hline & SWP45572 & 19:02:56 & 100 & 8874.328 \\
\hline & LWP23877 & $20: 53: 37$ & 30 & 8874.381 \\
\hline & SWP 45573 & $21: 33: 38$ & 74 & 8874.424 \\
\hline \multirow[t]{3}{*}{9 Sep. } & LWP23888 & 20:04:23 & 30 & 8875.347 \\
\hline & SWP45586 & $20: 42: 53$ & 100 & 8875.398 \\
\hline & LWP23889 & $22: 28: 15$ & 18 & 8875.443 \\
\hline \multirow[t]{3}{*}{10 Sep. } & SWP45595 & $19: 47: 17$ & 30 & 8876.335 \\
\hline & LWP23898 & $20: 33: 49$ & 100 & 8876.392 \\
\hline & SWP45596 & 22:19:30 & 28 & 8876.440 \\
\hline \multirow[t]{3}{*}{11 Sep. } & SWP45599 & 19:54:49 & 30 & 8877.340 \\
\hline & LWP23907 & $20: 32: 13$ & 100 & 8877.390 \\
\hline & SWP45600 & 22:19:41 & 28 & 8877.440 \\
\hline \multirow[t]{3}{*}{12 Sep. } & SWP45617 & 20:00:03 & 30 & 8878.344 \\
\hline & LWP23912 & $20: 35: 24$ & 60 & 8878.379 \\
\hline & SWP45618 & $21: 50: 47$ & 60 & 8878.431 \\
\hline \multirow[t]{3}{*}{13 Sep. } & SWP45625 & 20:05:19 & 30 & 8879.347 \\
\hline & LWP23923 & $20: 51: 21$ & 60 & 8879.390 \\
\hline & SWP45626 & $21: 58: 12$ & 50 & 8879.433 \\
\hline \multirow[t]{3}{*}{14 Sep. } & SWP45642 & $19: 23: 44$ & 30 & 8880.319 \\
\hline & LWP23938 & 20:06:15 & 60 & 8880.359 \\
\hline & SWP45643 & $21: 15: 08$ & 94 & 8880.418 \\
\hline \multirow[t]{3}{*}{15 Sep. } & SWP45651 & 19:01:52 & 30 & 8881.303 \\
\hline & LWP23945 & $20: 41: 13$ & 60 & 8881.383 \\
\hline & SWP45652 & $21: 49: 48$ & 60 & 8881.430 \\
\hline \multirow[t]{3}{*}{16 Sep. } & SWP 45660 & 19:58:31 & 30 & 8882.343 \\
\hline & LWP23958 & $20: 43: 58$ & 60 & 8882.385 \\
\hline & SWP45661 & $21: 52: 25$ & 55 & 8882.430 \\
\hline
\end{tabular}

vation and vice versa. A log of the spectra will be found in Table 6.

\subsection{Microwave observations}

II Peg was also monitored between 7-15 September 1992 using the Broad-band Interferometer (BBI) (Padin et al. 1987) at the Nuffield Radio Astronomy Laboratory (NRAL) at Jodrell Bank, England. The BBI operates at 
$5 \mathrm{GHz}(\lambda=6 \mathrm{~cm})$ with a bandwidth of $384 \mathrm{MHz}$ and has a sensitivity of $2 \mathrm{mJy} \min ^{-\frac{1}{2}}$.

The observations consisted of single scans of the source, each nominally $45 \mathrm{~min}$ but, due to telescope tracking errors (due, in turn, to high winds), actually achieved a typical on-source integration time of $20-40 \mathrm{~min}$. Individual integrations each lasted $20 \mathrm{~s}$. After averaging these $20 \mathrm{~s}$ integrations over a scan the typical noise level was $\sim 0.3-1.0 \mathrm{mJy}$.

The BBI was on $24 \mathrm{hr}$ scheduled operation observing a number of objects including II Peg. Amplitude calibrators, $3 \mathrm{C} 48$ and $3 \mathrm{C} 286$, were observed every $\sim 12 \mathrm{hr}$. Their flux densities were taken as 5.335 Jy and 7.338 Jy respectively (Baars et al. 1977). A nearby object, $2004+403$, was chosen as a phase calibrator with an observing cycle of 15 minutes on phase calibrator and 45 minutes on II Peg (referred to as a scan). A tracking window of 3 arcmin was imposed which rejected the incoming data when the observing source fell outside this window due to tracking error.

Details of the coverage achieved will be found in Table 7.

\section{Results}

\subsection{Photometry}

\subsubsection{Standard photometry}

A comparison of the mean magnitudes and colours of the comparison star SAO 91568 in Sect. 2.1 above shows that, for the three ground-based sets of photometry, zero points are reasonably consistent. The differences indicated by this comparison suggest mean differences as follows:

$\mathrm{SO}-\mathrm{JKT}: \Delta V=+0.017 ; \Delta(B-V)=+0.030 ; \Delta(U-$ $B)=-0.021$

SO - BNO: $\Delta V=-0.015 ; \Delta(B-V)=-0.009 ; \Delta(U-$ $B)=-0.037$.

No comparison was possible for the $(V-R)_{\mathrm{C}}$ and $(V-I)_{\mathrm{C}}$ data since these were measured at the JKT only. These differences in the magnitudes and colours of SAO 91568 are within the errors expected in the three data sets and so are unlikely to indicate systematic differences.

Intercomparison of the mean results for SAO 91577, however, indicates a large discrepancy between SO and $\mathrm{JKT}$ in respect of $(U-B)$ colour $\left(\Delta(U-B)_{\mathrm{SO}-\mathrm{JKT}}=\right.$ $-0.181)$. This is uncomfortably large to be attributed to photometric errors. It is possible, of course, that the bluer photometric transformations, particularly $U$, are uncertain at the extreme red end of their range. On the other hand the mean $(U-B)$ colours for II Peg itself are in good agreement (cf. Fig. 1). At present this anomaly is unresolved.

The $V$ light curve, as presented in Fig. 1, is a superposition of the data from all three sources. Its scatter
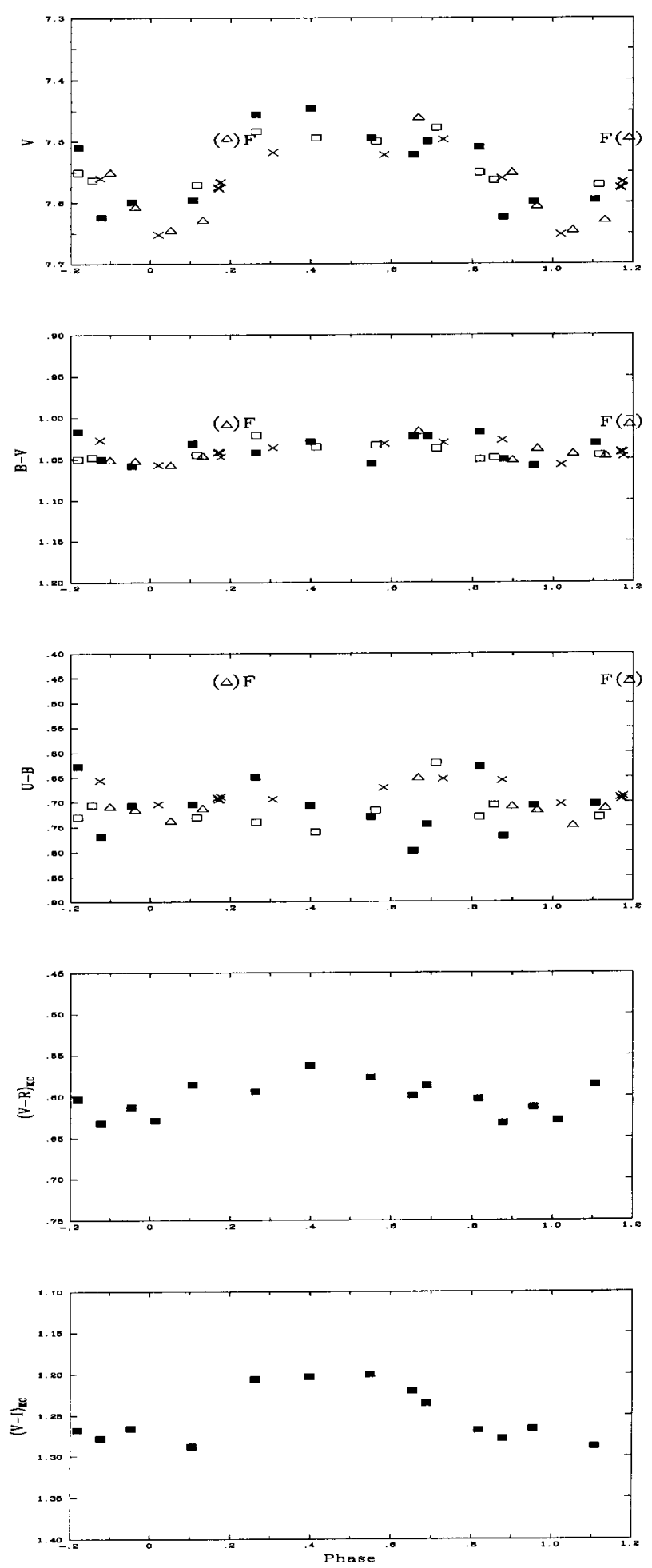

Fig. 1. Nightly mean $V$ and $(B-V),(U-B),(V-R)_{\mathrm{C}}$ and $(V-I)_{\mathrm{C}}$ light curves for II Peg in September 1992. The filled squares represent data from JKT in September, the open squares those from Stephanion Observatory in July, the open triangles those from the same source in September and the stars those from the Bulgarian National Observatory. The point bracketted and labelled " $F$ " is that measured during the flare of 12 September 
Table 7. Log of the BBI $5 \mathrm{GHz}$ monitoring of II Peg obtained between 7-15 September 1992. Phases have been calculated according to the ephemeris of Vogt (1981)

\begin{tabular}{|c|c|c|c|c|c|c|c|c|c|}
\hline $\begin{array}{l}\text { Day } \\
1992\end{array}$ & $\mathrm{UT}$ & $\begin{array}{c}\text { JD } \\
(\text { mid-exp) } \\
2440000.0+\end{array}$ & $\begin{array}{l}\text { Flux } \\
\text { mJy }\end{array}$ & $\begin{array}{c}\sigma \\
\mathrm{mJy}\end{array}$ & $\begin{array}{l}\text { Day } \\
1992\end{array}$ & $\mathrm{UT}$ & $\begin{array}{c}\text { JD (mid-exp) } \\
2440000.0+\end{array}$ & $\begin{array}{l}\text { Flux } \\
\text { mJy }\end{array}$ & $\begin{array}{c}\sigma \\
\mathrm{mJy}\end{array}$ \\
\hline 7 Sep. & $17: 35-18: 00$ & 8873.241 & 2.5 & 0.5 & 10 Sep. & $18: 18-19: 00$ & 8876.277 & 1.111 & 0.4 \\
\hline 7 Sep. & $18: 24-19: 00$ & 8873.279 & 2.7 & 0.5 & 10 Sep. & $19: 19-20: 00$ & 8876.319 & 2.584 & 0.5 \\
\hline 7 Sep. & $19: 19-20: 00$ & 8873.319 & 1.9 & 0.4 & 10 Sep. & $20: 19-21: 00$ & 8876.361 & 2.485 & 0.5 \\
\hline 7 Sep. & $20: 18-21: 00$ & 8873.360 & 2.0 & 0.5 & 10 Sep. & $21: 19-21: 56$ & 8876.401 & 3.524 & 0.6 \\
\hline 7 Sep. & $21: 18-22: 00$ & 8873.402 & 4.9 & 0.5 & 10 Sep. & $22: 19-23: 00$ & 8876.444 & 3.718 & 1.1 \\
\hline 7 Sep. & $22: 18-22: 59$ & 8873.443 & 1.8 & 0.5 & 10 Sep. & $23: 31-23: 59$ & 8876.490 & 4.797 & 1.0 \\
\hline 7 Sep. & $23: 23-23: 59$ & 8873.487 & 3.4 & 0.7 & & & & & \\
\hline 8 Sep. & $00: 20-00: 58$ & 8873.527 & 5.1 & 0.6 & 11 Sep. & $19: 18-20: 00$ & 8877.319 & 3.036 & 0.5 \\
\hline 8 Sep. & $01: 20-02: 00$ & 8873.569 & 3.8 & 0.5 & 11 Sep. & $20: 18-21: 00$ & 8877.360 & 2.176 & 0.4 \\
\hline 8 Sep. & $02: 20-03: 00$ & 8873.611 & 5.7 & 0.6 & $\begin{array}{l}11 \text { Sep. } \\
11 \text { Sep. }\end{array}$ & $\begin{array}{l}21: 20-22: 00 \\
22: 25-22: 59\end{array}$ & $\begin{array}{l}8877.403 \\
8877.446\end{array}$ & $\begin{array}{c}0.903 \\
1.1\end{array}$ & $\begin{array}{l}0.4 \\
0.6\end{array}$ \\
\hline 8 Sep. & $17: 11-18: 00$ & 8874.233 & 1.4 & 0.4 & 11 Sep. & $23: 21-23: 59$ & 8877.486 & 3.851 & 0.8 \\
\hline 8 Sep. & $18: 18-19: 00$ & 8874.277 & 2.3 & 0.4 & & & & & \\
\hline 8 Sep. & $19: 18-20: 00$ & 8874.319 & 1.6 & 0.4 & 12 Sep. & $19: 18-20: 00$ & 8878.319 & 3.331 & 0.5 \\
\hline 8 Sep. & $20: 18-21: 00$ & 8874.360 & 2.1 & 0.5 & 12 Sep. & $20: 18-21: 00$ & 8878.360 & 2.120 & 0.4 \\
\hline 8 Sep. & $21: 22-21: 38$ & 8874.396 & 1.0 & 0.7 & 12 Sep. & $21: 19-22: 00$ & 8878.403 & 0.248 & 0.5 \\
\hline 8 Sep. & $22: 19-22: 59$ & 8874.444 & 2.4 & 0.5 & 12 Sep. & $22: 19-23: 00$ & 8878.446 & 0.437 & 0.7 \\
\hline 8 Sep. & $23: 19-23: 58$ & 8874.485 & 5.2 & 0.8 & 12 Sep. & $23: 19-23: 58$ & 8878.486 & 1.739 & 0.8 \\
\hline 9 Sep. & 00:19-01:00 & 8874.527 & 5.9 & 0.6 & & & & & \\
\hline 9 Sep. & $01: 20-01: 59$ & 8874.569 & 3.0 & 0.5 & 13 Sep. & $19: 18-20: 00$ & 8879.319 & 11.159 & 0.9 \\
\hline 9 Sep. & $02: 20-03: 00$ & 8874.611 & 4.9 & 0.6 & 13 Sep. & $20: 19-21: 00$ & 8879.361 & 12.901 & 1.0 \\
\hline 9 Sep. & $03: 19-04: 00$ & 8874.652 & 4.7 & 0.5 & 13 Sep. & $21: 20-22: 00$ & 8879.403 & 14.976 & 1.1 \\
\hline 9 Sep. & $04: 19-05: 00$ & 8874.694 & 3.4 & 0.5 & $\begin{array}{l}13 \text { Sep. } \\
13 \text { Sep. }\end{array}$ & $\begin{array}{l}22: 19-23: 00 \\
23: 19-23: 59\end{array}$ & $\begin{array}{l}8879.444 \\
8879.485\end{array}$ & $\begin{array}{c}9.523 \\
9.1\end{array}$ & $\begin{array}{l}0.9 \\
0.9\end{array}$ \\
\hline 9 Sep. & $18: 38-18: 59$ & 8875.280 & 1.4 & 0.7 & & & & & \\
\hline 9 Sep. & $19: 18-20: 00$ & 8875.319 & 0.2 & 0.4 & 14 Sep. & $19: 24-19: 59$ & 8880.320 & 5.181 & 0.6 \\
\hline 9 Sep. & $20: 18-21: 00$ & 8875.360 & 1.4 & 0.5 & 14 Sep. & $20: 19-21: 00$ & 8880.361 & 2.769 & 0.5 \\
\hline 9 Sep. & $21: 20-22: 00$ & 8875.403 & 0.3 & 0.5 & 14 Sep. & $21: 18-22: 00$ & 8880.402 & 5.245 & 0.6 \\
\hline 9 Sep. & $22: 20-23: 00$ & 8875.444 & 1.0 & 0.5 & 14 Sep. & $22: 18-22: 59$ & 8880.443 & 2.169 & 0.7 \\
\hline 9 Sep. & $23: 29-23: 56$ & 8875.488 & 3.7 & 1.1 & 14 Sep. & $23: 22-23: 59$ & 8880.486 & 1.923 & 0.6 \\
\hline 10 Sep. & $00: 19-00: 59$ & 8875.527 & 3.1 & 0.6 & & & & & \\
\hline 10 Sep. & $01: 20-02: 00$ & 8875.569 & 2.5 & 0.6 & 15 Sep. & $19: 20-19: 58$ & 8881.319 & 1.992 & 0.6 \\
\hline 10 Sep. & $02: 20-03: 00$ & 8875.611 & 2.8 & 0.5 & 15 Sep. & $20: 24-21: 00$ & 8881.363 & 0.990 & 0.9 \\
\hline 10 Sep. & 03:19-04:00 & 8875.652 & 2.2 & 0.4 & 15 Sep. & $21: 19-22: 00$ & 8881.402 & 0.607 & 0.7 \\
\hline 10 Sep. & 04:19-05:00 & 8875.694 & 1.1 & 0.4 & 15 Sep. & $22: 20-22: 57$ & 8881.443 & 2.485 & 0.9 \\
\hline & & & & & 15 Sep. & $23: 20-24: 00$ & 8881.486 & 0.7 & 0.6 \\
\hline
\end{tabular}

is larger than might be expected from the intercomparison of the mean magnitudes for SAO 91568. It can, nevertheless, be readily seen that there is a minimum near phase $\varphi \sim 0.03$ and a maximum near $\varphi \sim 0.4$. Thus the light curve is clearly asymmetric, with a much steeper rise than fall. However, there is a relatively slow fall between $\varphi \sim 0.4-0.8$, followed by a much more rapid decline to minimum. The amplitude of the modulation, $\Delta V$ is $\sim 0.2$.

The scatter in the $B-V$ curve is reasonably uniform with phase and is of amplitude $\Delta(B-V) \sim 0.02-0.03$. Overall, the $B-V$ curve shows evidence of a low-level modulation $(\Delta(B-V) \sim 0.02)$ in phase with the $V$ variation.

The $U-B$ curve shows a uniform scatter with amplitude of $\Delta(U-B) \sim 0.15-0.2$ apart from one point which stands out from the rest (near $\varphi \sim 0.2$ ). This is associated with the large optical flare on 5 September which will be discussed further below (Sect. 3.2). It is indicated in both the $U-B$ and $B-V$ colour curves and in the $V$ light curve by an "F". We note that the scatter in the $(U-B)$ measurements is much larger than would be expected on the basis of the uncertainty in the measurements themselves (i.e. $~ 0.03$ ). This may be due to low-level flaring as 

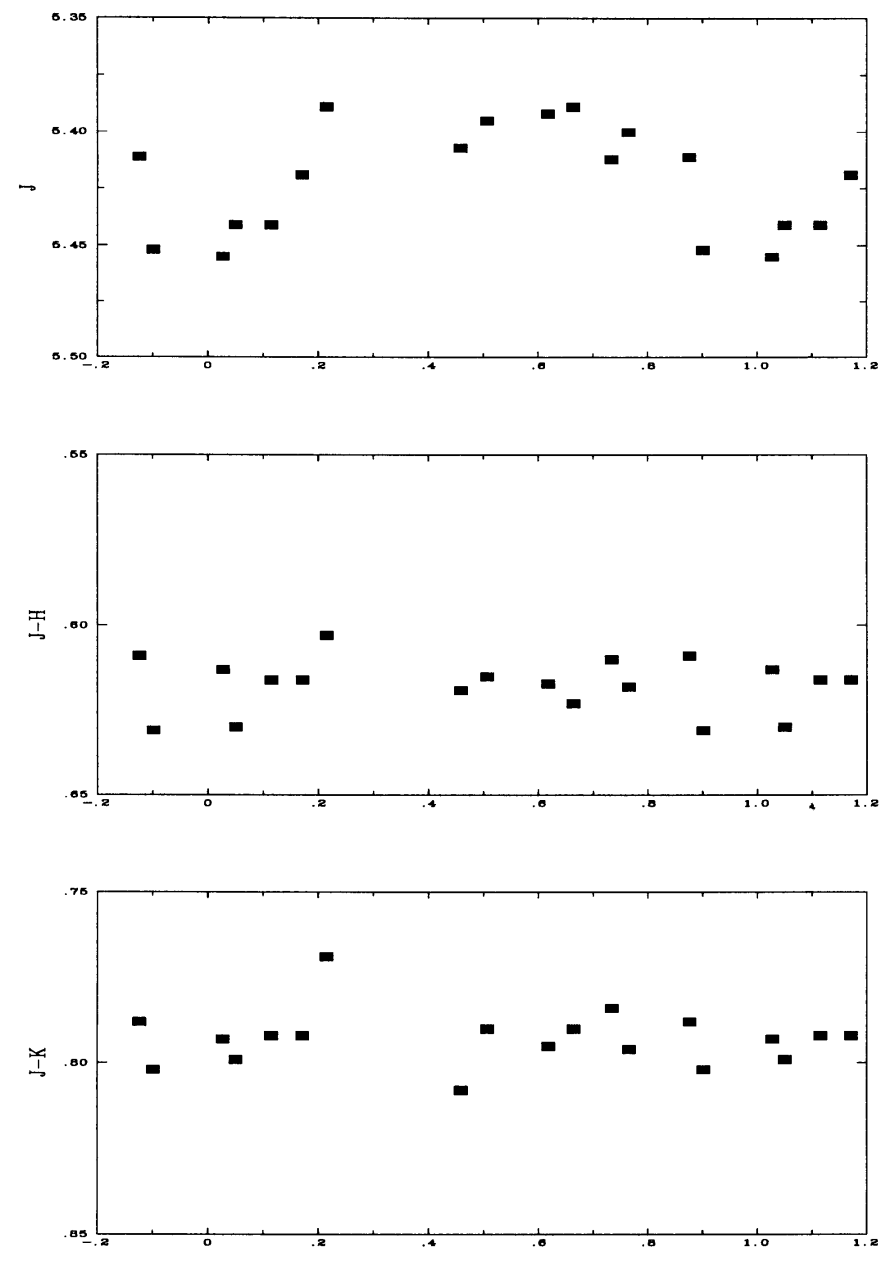

Fig. 2. The $J$ magnitude and $(J-H)$ and $(J-K)$ colour curves for II Peg at the end of July and the first half of August 1992 as observed at the Teide Observatory of the IAC. Phases have been calculated according to the ephemeris of Vogt (1981), i.e. $\mathrm{JD}=2443033.47+6.72422 E$

has been suggested in many active late-type stars (see e.g. Byrne 1983 and references therein).

Both the near-IR colour curves mirror the $V$ variation accurately in phase. $V-R$, however, has an amplitude of only $\Delta(V-R) \sim 0.07$, while $\Delta(V-I) \sim 0.11$. Both are again in phase with $V$, consistent in a general sense, with the spot origin of the variations.

The light curves for the IR $J H K$ bands will be found in Fig. 2 in the form of $J$ magnitude and $(J-H)$ and $(J-K)$ colour diagrams. The $J$ light curve is similar to $V$ but with an amplitude of $\Delta J \sim 0.06$. There is no evidence of systematic variation in either of the IR colours themselves, but, given the different amplitudes in $V$ and $J$, there is a strong variation in $(V-J)$. Because, however, simultaneous $V$ and $J H K$ measurements are not available, it is not possible to get a direct measure of the $(V-J)$ colour.

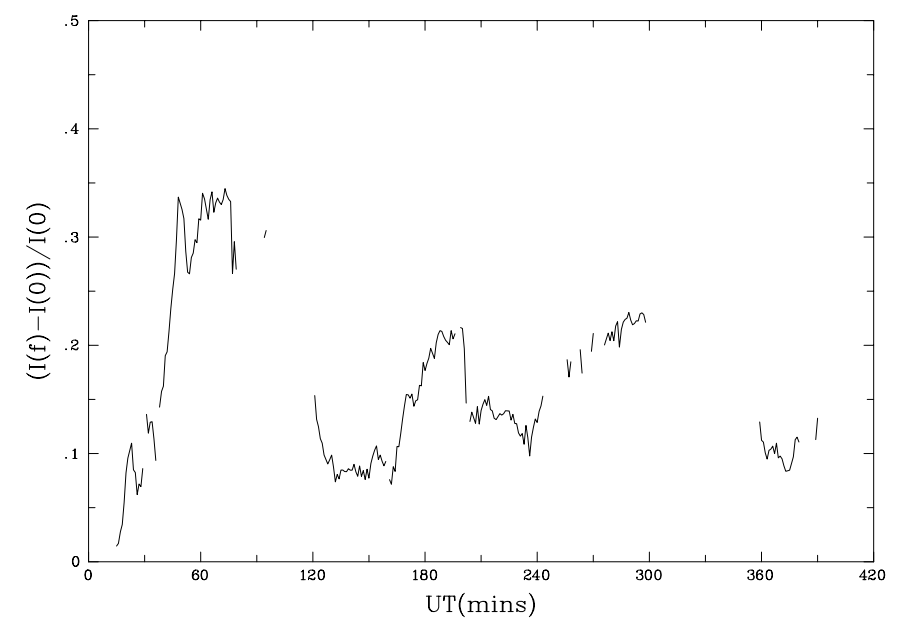

Fig. 3. Light curve of the large optical flare observed in the $U$-band at Stephanion Observatory on 5 September 1992

\subsection{U-band flare monitoring}

Two optical $U$-band flares were recorded, details of which will be found in Table 8 . The light curve of the largest of these will be found in Fig. 3 .

Table 8. Details of the two optical flares recorded in Johnson $U$ on II Peg at Stephanion Observatory. See Sect. 3.2 for an explanation of the derivation of the total integrated energy, $E_{U}$, of each flare

\begin{tabular}{|c|c|c|c|c|}
\hline Date & UT & $\overline{\Delta \mathrm{U}}$ & $E D$ & $E_{U}$ \\
\hline 1992 & $\max$ & & $\mathrm{s}$ & erg \\
\hline 05 Sep. & 20:39 & $0.32^{*}$ & $>3925$ & $>1.7510^{35}$ \\
\hline 12 Sep. & 21:49 & 0.29 & 59 & $2.6310^{33}$ \\
\hline
\end{tabular}

${ }^{*}$ This flare is complex (see Fig. 3).

We have used the Equivalent Duration $(E D)$ method of Gershberg (1972) in calculating the total, timeintegrated energy in each flare. $E D$ is defined as the time during which the quiescent star emits the same energy as the flare in the same passband. Thus the measurement of the flare's energy is referred to the quiescent pre-flare level as measured immediately preceding the flare itself. This avoids many of the uncertainties involved in the absolute calibration of the flare observations.

The energy of the flare is thus defined as

$E_{U}=E D \times F_{\mathrm{q}, U}$

where $F_{\mathrm{q}, U}$ is the quiescent energy emitted in the $U$ band per second. This, in turn, is related to the star's quiescent $U$ magnitude (taken as 9.3 , see Table 1 ) by

$F_{\mathrm{q}, U}=4 \pi d^{2} 10^{-0.4 U} \Pi_{U}$

where $d$ is the distance to the star, taken as $29 \mathrm{pc}$ 
(Strassmeier et al. 1993), and $\Pi_{U}$ is the conversion factor from $U$ magnitude to energy, taken as $2.3210^{-6} \mathrm{erg} \mathrm{cm}^{-2} \mathrm{~s}^{-1}$ (Bessell 1979). This yields $F_{\mathrm{q}, U}=$ $4.4510^{31} \mathrm{erg} \mathrm{cm}^{-2} \mathrm{~s}^{-1}$. Deriving the energy of each flare in absolute terms is then a relatively simple procedure, the results of which will be found in Table 8 .

\subsection{High-resolution $H \alpha$}

The McMath high-resolution spectra were flat-fielded, and wavelength calibrated using purpose built routines within the IDL data analysis package (IDL Users' Guide, 1985). All subsequent analysis was undertaken using routines within the IRAF astronomical software suite (Tody et al. 1986) or within DIPSO (Howarth \& Murray 1987), a software package available on the UK STARLINK network (Bromage 1984). Spectra were corrected for telluric lines by reference to spectra of early-type stars taken specially for this purpose.

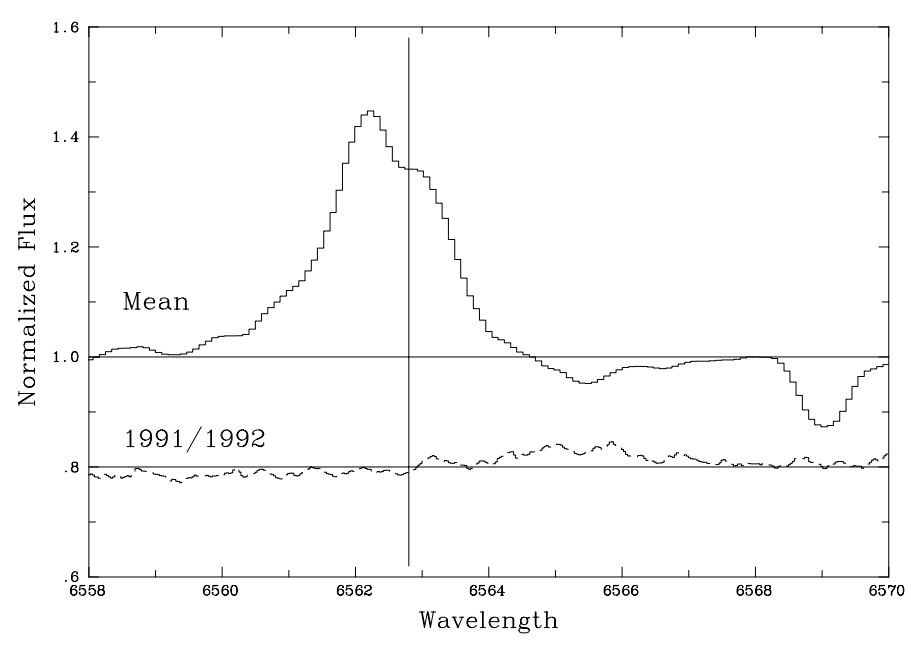

Fig. 4. The overall mean profile of II Peg's $\mathrm{H} \alpha$ emission line derived from four nights' data (upper solid curve). Note that all spectra have been wavelength corrected to the rest frame of the K-star primary and the vertical line gives the rest wavelength of the $\mathrm{H} \alpha$ line. The dashed curve gives the ratio of the mean 1992 spectrum to that for 1991 taken from Paper I and displaced downward to aid visibility. Both spectra have been smoothed by a gaussian of FWHM $\sim 0.18 \AA$, less than the nominal resolution of the spectrograph

The resulting spectra were then corrected to the rest frame of the $\mathrm{K}$ star primary of II Peg. This was achieved by shifting a strong, isolated photospheric line (FeI $\lambda 6569.224 \AA)$ to its laboratory wavelength. The spectra were then normalized by fitting a spline function to the local continuum and dividing through by this spline. The points at which the spline was fit were those judged to be free of lines when comparison was made with spectra of a number of slowly rotating $\mathrm{K}$ giants, taken with the same instrument and broadened to II Peg's rotational

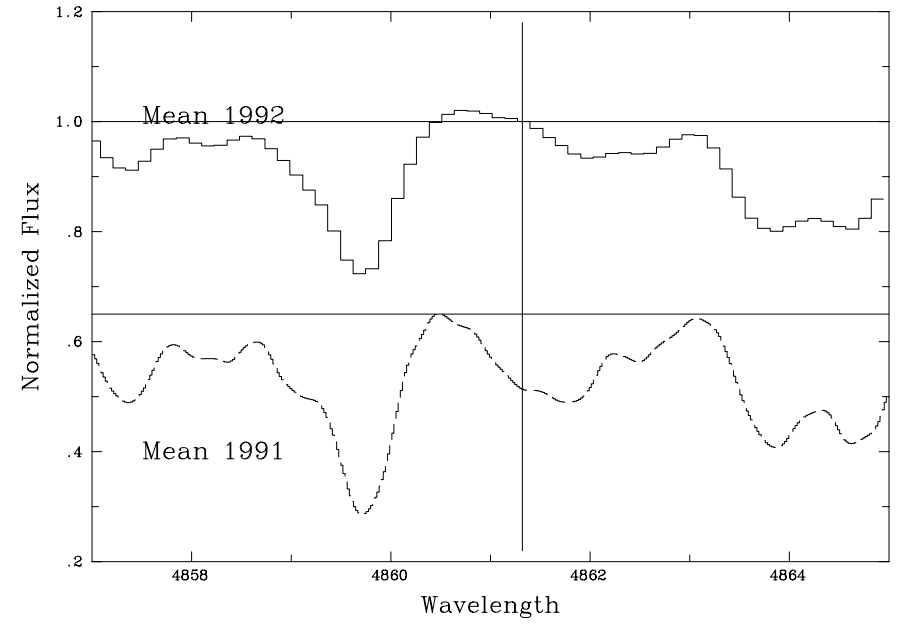

Fig. 5. The overall mean profile of II Peg's $\mathrm{H} \beta$ emission line derived from all three nights' data (upper curve). The dashed curve gives the mean $1991 \mathrm{H} \beta$ profile from Paper I which have been displaced downward to aid visibility. Note that all spectra have been wavelength corrected to the rest frame of the K-star primary and the vertical line gives the rest wavelength of the $\mathrm{H} \beta$ line

$v \sin i\left(=21 \mathrm{~km} \mathrm{~s}^{-1}, \operatorname{Vogt}(1981)\right)$. Figure 4 shows the mean of all the $\mathrm{H} \alpha$ spectra.

From Fig. 4 it will be seen that the mean $\mathrm{H} \alpha$ line is strongly in emission with a peak intensity $\sim 1.45$ times that of the local continuum and has a FWHM $\sim 1.6 \AA$. Furthermore, it is asymmetric, in the sense that it shows an "absorption reversal" whose red peak is depressed relative to the blue peak, and a blue wing which exceeds the red in total flux.

\subsection{High-resolution $H \beta$}

The McMath high-resolution spectra in the vicinity of the chromospheric $\mathrm{H} \beta$ emission line were extracted and analyzed similarly to those at $\mathrm{H} \alpha$ (Sect. 3.3). They are shown after correction to the rest frame of the K-star primary in Fig. 5 in the form of the overall mean spectrum derived from the data on all three nights of observation.

As remarked in Paper I the line is blended with nearby photospheric lines of $\mathrm{CrI} \lambda 4861.849 \AA$ and FeI $\lambda 4861.952 \AA$ on the red side and FeI $\lambda 4860.986 \AA$ on the blue. It is possible to "see" the lines to the red but the line to the blue is inextricably blended with $\mathrm{H} \beta$ and impossible to deblend without recourse to either synthetic spectra or inactive templates. However, it is apparent that the $\mathrm{H} \beta$ line is "filled in" and is asymmetric to the blue, in the sense that it is more "filled in" on that side of line centre.

\subsection{High-resolution $\mathrm{HeI} \mathrm{D}_{3}$}

The McMath high-resolution spectra in the vicinity of the chromospheric $\mathrm{HeI} \mathrm{D}_{3}$ line were extracted and analyzed 


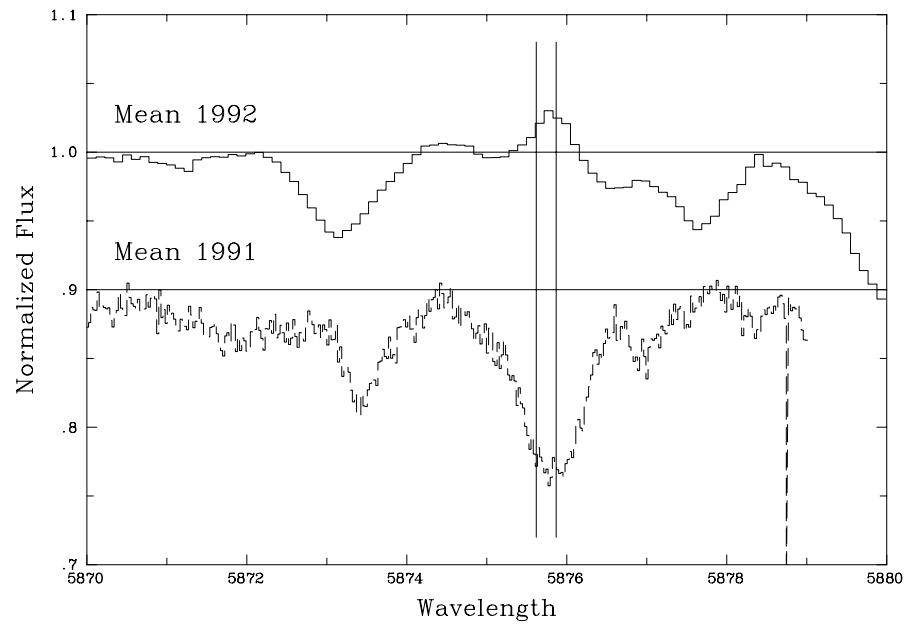

Fig. 6. The overall mean profile of II Peg's HeI $\mathrm{D}_{3}$ line derived from three nights' data (upper curve). The dashed curve gives the mean $1991 \mathrm{HeI} \mathrm{D}_{3}$ profile from Paper I which have been displaced downward to aid visibility. Note that all spectra have been wavelength corrected to the rest frame of the K-star primary and the vertical lines give the rest wavelength of the $\mathrm{HeI}_{3}$ doublet

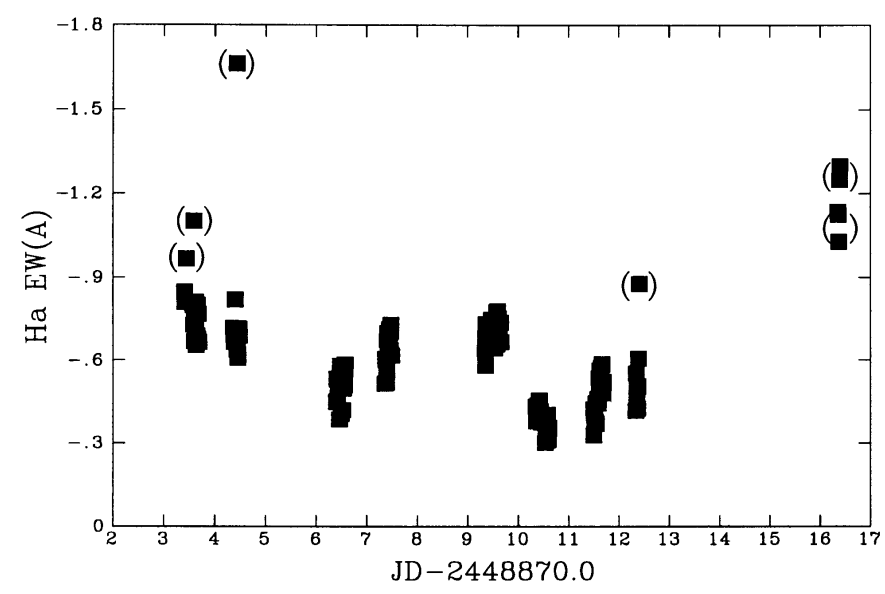

Fig. 7. The EW of the II Peg's $\mathrm{H} \alpha$ emission as a function of Julian Date measured at the Univeristy of Birmingham's Wast Hills Observatory between 7-20 September 1992. Proposed flares are indicated by bracketts around the flaring points

as were those for $\mathrm{H} \alpha$ (Sect. 3.3). The resultant spectra are shown in Fig. 6 in the form of the overall mean spectrum derived from the data on both nights of observation. The line is in net weak emission with a peak intensity of $\sim 2-3 \%$ of the continuum level.

\subsection{Low-resolution $H \alpha$}

These spectra were extracted in hardware in real-time at the telescope to give 1-dimensional data, which were later debiased, flat-fielded and wavelength calibrated. The re-
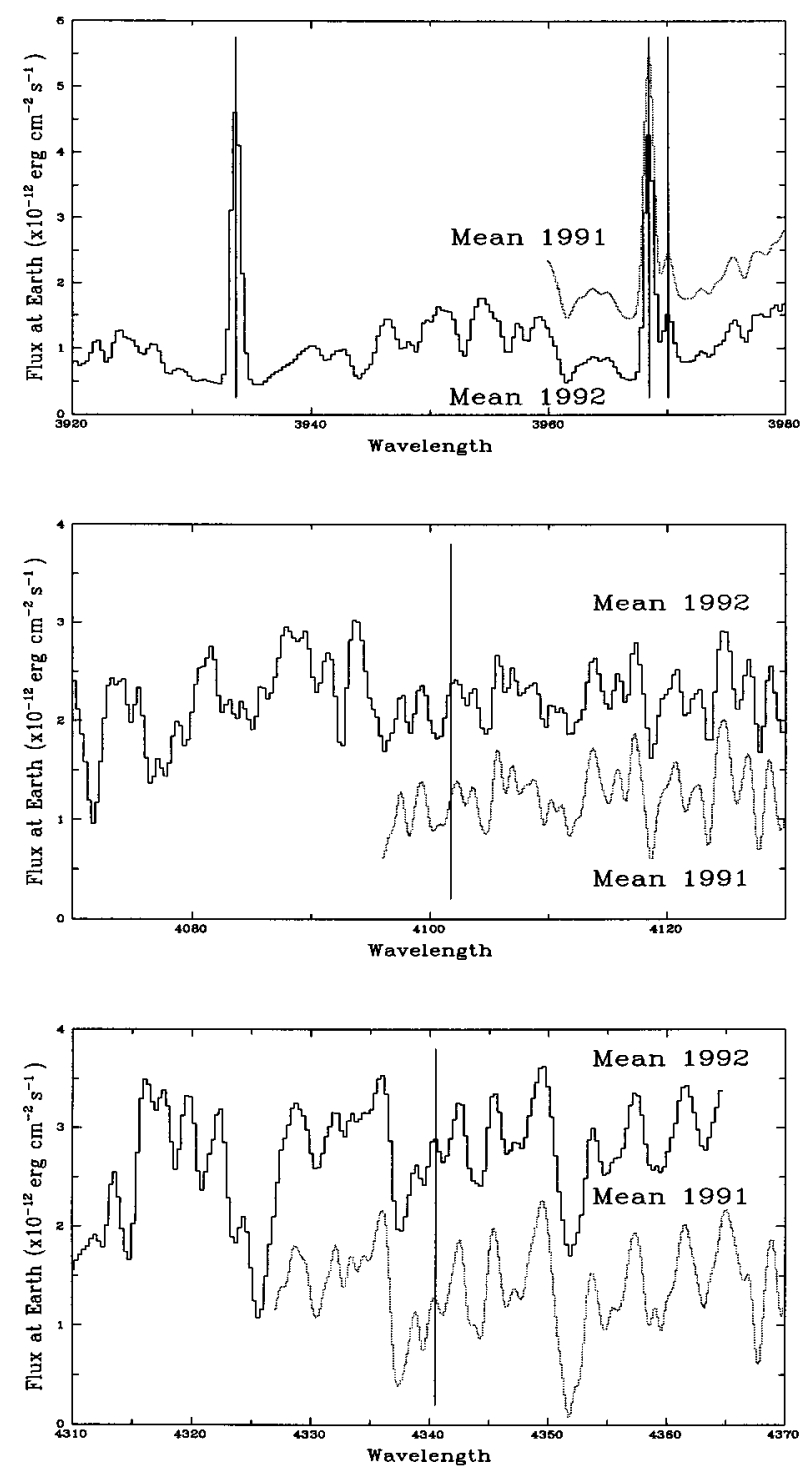

Fig. 8. Blue region spectra of II Peg taken at the Isaac Newton Telescope 14-19 September 1992 shifted to the rest frame of the $\mathrm{K}$ star. The top panel shows the five clear nights' spectra in the region of the CaII $H \& K$ lines as the overall mean spectrum. The middle and lower panels give the same data in the vicinity of the Balmer $\mathrm{H} \delta$ and the Balmer $\mathrm{H} \gamma$ lines. Also shown are spectra in these same spectral regions taken in 1991 (Paper I) smoothed to match the spectral resolution of the present data (dashed curves). The vertical lines indicate the rest wavelengths of the Balmer and CaII lines

sults of the low-resolution $\mathrm{H} \alpha$ monitoring will be found in Fig. 7 as a plot of $\mathrm{EW}(\mathrm{H} \alpha)$ against time.

\subsection{Blue low-resolution spectroscopy}

The INT spectra were extracted from the CCD images and wavelength calibrated within the STARLINK package FIGARO (Meyerdicks 1993). We illustrate the results in Figs. 8 where the overall mean spectrum has been shifted 
in wavelength to match the rest frame of the $\mathrm{K}$ star. A flux calibration was achieved by reference to measurements of flux standards before and after the exposures. Although these spectra were not spectrophotometric, intercomparison between flux standards suggests that the accuracy of this calibration is better than $\sim 20 \%$ in all cases.

We note the following general characteristics. The CaII $H \& K$ and Balmer $\mathrm{H} \epsilon$ lines are strongly in emission. However, we have examined carefully the spectra in the region of the Balmer $\mathrm{H} \gamma$ or $\mathrm{H} \delta$ lines (marked by vertical lines in Figs. 8) and find that there is no obvious evidence of either, whether in emission or absorption.

\section{8. $U V$ spectroscopy}

The IUE spectra were extracted from the spectral images and then wavelength and flux calibrated using the program IUEDR (Giddings 1983) which is available on the UK STARLINK astronomical computing network (Bromage 1984). Subsequent analysis was performed using the STARLINK program DIPSO (Howarth \& Murray 1987).

\subsubsection{SWP}

In the SWP spectra the stellar lines are unresolved and their fluxes were estimated by fitting one or two gaussian profiles to the data in the manner described in Byrne et al. (1987). Two gaussians of fixed separation and relative intensity were used where the lines were partially resolved doublets (e.g. CIV $\lambda 1549 / 52 \AA$ ). The line fluxes for the most prominent SWP emission lines resulting from this procedure will be found tabulated in Tables 9 .

\subsubsection{LWP}

Extracting line fluxes from the LWP spectra was more complex for a number of reasons. The most prominent lines visible in the LWP spectra were those of the MgII $h \& k$ resonance doublet $(\lambda 2795.5 / 2802.7 \AA)$ and some lines of the FeII UV1 multiplet near $\lambda 2600 \AA$. In both cases the emission lines were resolved. Furthermore, in the case of the MgII $h \& k$ resonance doublet there is appreciable interstellar (IS) absorption superimposed on the stronger and broader stellar emission. Finally, the wavelength calibration of IUE in HIRES may be subject to uncertainties of order $\approx \pm 10 \mathrm{~km} \mathrm{~s}^{-1}$ (Byrne et al. 1989).

We have adopted the following procedure for deriving the flux of the MgII lines. We have assumed that the IS line is unresolved at the resolution of IUE HIRES and fitted it with a gaussian profile of fixed FWHM equal to that of the instrumental profile of the IUE spectrograph in HIRES mode, while simultaneously fitting the main emission profile with another gaussian whose central wavelength, FWHM and intensity are all free to vary. This procedure fits the IS line well but it is clear that there is excess flux in the wings of the main stellar emission line over and above a gaussian.

After this first round of fits the IS feature was used as a fiducial to bring the entire set of spectra to a common wavelength scale and the stellar emission fitted with the sum of two gaussians, one to represent the main body of the emission and the other to represent the wings. These fits have been used to estimate the total flux in the stellar emission line. The result will be found in Table 10 .

The FeII lines were measured by fitting a number of gaussians of fixed wavelength separation, corresponding to the laboratory separation of the UV1 lines. Their FWHM and intensities were, on the other hand, allowed to vary freely. The resulting measured line fluxes will also be found in Table 10.

\subsection{Microwave observations}

The data from each scan was vector integrated over the whole scan period and a plot of these scan averages will be found in Fig. 9. The star is detected at all times of observation and shows evidence of continuous variability in its flux at $5 \mathrm{GHz}$ at all time scales examined by the data. This is true both on an hourly time scale and from night-to-night. A strong flare with a peak flux density of 15 mJy was observed on September 13.

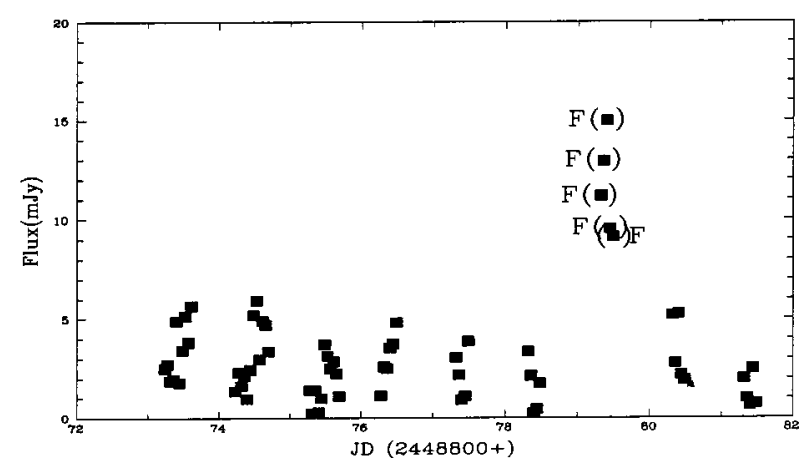

Fig. 9. The time sequence of BBI $5 \mathrm{GHz}$ observations of II Peg. The flaring points discussed in the text are labelled with an "F"

\section{Discussion}

\subsection{Mean light curve}

The mean $V$ magnitude in 1991 (Paper I), $\langle V>\sim 7.45$, was clearly brighter than the current epoch, $\langle V>\sim 7.55$. Our measured mean is, however, very similar to those measured in 1986 (Byrne 1986; Byrne \& Marang 1987), $<V>\sim 7.53$, and 1989 (Doyle et al. 1992a), $\langle V>\sim$ 7.54, when II Peg showed a record large amplitude modulation. Assuming that the fainter mean magnitude is caused by a relatively larger global coverage by starspots, 
Table 9. Line fluxes at Earth for the most prominent emission lines in the SWP spectra of II Peg. Values given in bold type were obtained from spectra during which we have deemed flares to be taking place. The phase of each observation is given according to the ephemeris of Vogt (1981), i.e. $\mathrm{JD}=2443033.47+6.72422 E$

\begin{tabular}{|c|c|c|c|c|c|c|c|c|c|}
\hline $\begin{array}{c}\text { JD } \\
\text { mid-exp } \\
2440000.0+\end{array}$ & Phase & $\begin{array}{c}\text { OI } \\
\lambda 1302 / 5 / 6 \AA\end{array}$ & $\begin{array}{c}\text { CII } \\
\lambda 1335 / 6 \AA\end{array}$ & $\begin{array}{c}\text { CIV } \\
\lambda 1548 / 51 \AA \\
\text { Line Flux at }\end{array}$ & $\begin{array}{c}\text { HeII } \\
\lambda 1640 \AA \\
\text { Larth }\left(\times 10^{-}\right.\end{array}$ & $\begin{array}{c}\text { CI } \\
\lambda 1656-58 \AA \\
\left.\mathrm{erg} \mathrm{cm}^{-2} \mathrm{~s}^{-1}\right)\end{array}$ & $\begin{array}{c}\text { AlII } \\
\lambda 1671 \AA\end{array}$ & \multicolumn{2}{|c|}{$\begin{array}{c}\text { SiII } \\
\lambda 1808 / 17 / 8 \AA\end{array}$} \\
\hline 8871.327 & 0.183 & 6.9 & 12.1 & 37.2 & 15.4 & 5.3 & 2.7 & 1.8 & 3.4 \\
\hline 8871.411 & 0.196 & 6.7 & 12.2 & 25.3 & 15.0 & 5.6 & 2.9 & 1.4 & 2.9 \\
\hline 8872.380 & 0.340 & 4.6 & 4.8 & 5.3 & 4.6 & 3.1 & 1.1 & 1.4 & 2.2 \\
\hline 8873.368 & 0.487 & 3.2 & 3.7 & 3.9 & 3.3 & 2.1 & 1.1 & 1.0 & 1.6 \\
\hline 8873.441 & 0.498 & 2.6 & 2.5 & 4.7 & 2.9 & 1.6 & 1.1 & 0.7 & 1.2 \\
\hline 8874.221 & 0.614 & 3.2 & 4.5 & 4.2 & 4.3 & 3.0 & 2.2 & 1.3 & 1.7 \\
\hline 8874.328 & 0.630 & 2.4 & 2.4 & 3.7 & 4.0 & 2.8 & 1.1 & 1.0 & 1.2 \\
\hline 8874.424 & 0.644 & 3.2 & 4.6 & 5.9 & 4.3 & 3.0 & 0.7 & 1.4 & 1.8 \\
\hline 8875.398 & 0.789 & 3.3 & 4.8 & 9.8 & 6.6 & 2.5 & 1.2 & 1.7 & 2.5 \\
\hline 8876.335 & 0.928 & 3.9 & 3.3 & 5.0 & 4.6 & 2.7 & 1.0 & 1.2 & 2.0 \\
\hline 8876.440 & 0.944 & 3.4 & 2.8 & 4.9 & 3.7 & 1.9 & - & 1.3 & 2.3 \\
\hline 8877.340 & 0.078 & 3.5 & 4.8 & 4.8 & 4.1 & 3.0 & 0.6 & 1.4 & 2.2 \\
\hline 8877.440 & 0.093 & 2.7 & 2.8 & 3.0 & 3.7 & 2.8 & 0.7 & 1.0 & 1.8 \\
\hline 8878.344 & 0.227 & 3.7 & 4.3 & 5.6 & 3.5 & 3.1 & - & 1.4 & 2.3 \\
\hline 8878.431 & 0.240 & 4.8 & 6.2 & 9.8 & 5.1 & 3.3 & 1.4 & 1.5 & 1.9 \\
\hline 8879.347 & 0.376 & 4.3 & 3.5 & 5.1 & 3.0 & 2.5 & 0.8 & 1.2 & 1.8 \\
\hline 8879.433 & 0.389 & 3.9 & 4.8 & 5.8 & 4.2 & 2.7 & 0.7 & 1.4 & 2.2 \\
\hline 8880.319 & 0.521 & 3.5 & 4.2 & 5.1 & 3.3 & 2.2 & 0.8 & 1.2 & 2.0 \\
\hline 8880.418 & 0.536 & 3.6 & 3.8 & 4.2 & 3.5 & 2.6 & 0.7 & 1.3 & 1.7 \\
\hline 8881.303 & 0.667 & 3.2 & 2.3 & 5.1 & 3.9 & 2.0 & 0.8 & 1.5 & 1.6 \\
\hline 8881.430 & 0.686 & 3.7 & 3.4 & 5.7 & 3.7 & 2.7 & - & 1.7 & 3.0 \\
\hline 8882.343 & 0.822 & 1.3 & 1.3 & 2.9 & 1.9 & 1.4 & - & 0.8 & 1.3 \\
\hline 8882.430 & 0.835 & 3.2 & 4.0 & 6.3 & 4.3 & 2.9 & - & 1.3 & 2.1 \\
\hline
\end{tabular}

Table 10. Line fluxes at Earth for the most prominent emission lines in the LWP spectra of II Peg. Note that the MgII line fluxes have been corrected for interstellar absorption. The values given in boldface are those associated with the flare discussed in the text. Phase has been calculated using the ephemeris of Vogt $(1981)$, i.e. JD $=2443033.47+6.72422 E$

\begin{tabular}{|c|c|c|c|c|c|c|c|c|}
\hline \multirow{3}{*}{$\begin{array}{c}\text { JD } \\
\text { mid-exp } \\
2440000.0+\end{array}$} & \multirow[t]{3}{*}{ Phase } & \multicolumn{2}{|c|}{ MgII } & \multicolumn{5}{|c|}{ Fe II } \\
\hline & & $2795.5 \AA$ & $2802.7 \AA$ & $2620.7 \AA$ & $2621.7 \AA$ & 2625.7 & $2628.3 \AA$ & $2631.1 \AA$ \\
\hline & & $\times 10^{-12} \mathrm{e}$ & $\mathrm{cm}^{-2} \mathrm{~s}^{-1}$ & & $\times 10$ & 13 erg c & & \\
\hline 8871.357 & 0.188 & 10.90 & 9.08 & 2.71 & 3.90 & 8.45 & 4.57 & 8.83 \\
\hline 8872.328 & 0.332 & 7.13 & 5.05 & 3.13 & 1.78 & 4.31 & 2.33 & 2.98 \\
\hline 8872.431 & 0.348 & 5.92 & 4.61 & 2.49 & 1.34 & 3.80 & 1.37 & 3.03 \\
\hline 8873.316 & 0.479 & 5.85 & 5.47 & 3.41 & 2.56 & 3.20 & 1.88 & 2.25 \\
\hline 8873.418 & 0.495 & 5.93 & 4.62 & 3.33 & 1.04 & 4.38 & 2.04 & 3.68 \\
\hline 8874.171 & 0.606 & 5.40 & 4.68 & 1.85 & 1.66 & 3.08 & 2.03 & 2.76 \\
\hline 8874.277 & 0.622 & 5.67 & 5.23 & 1.96 & 0.51 & 2.66 & 1.63 & 2.22 \\
\hline 8874.381 & 0.638 & 5.45 & 5.67 & 2.46 & 1.84 & 3.69 & 2.63 & 1.64 \\
\hline 8875.347 & 0.781 & 5.81 & 5.30 & 2.58 & 1.31 & 2.76 & 2.17 & 2.39 \\
\hline 8875.443 & 0.796 & 5.68 & 5.29 & 2.55 & 2.17 & 3.18 & 2.94 & 4.57 \\
\hline 8876.392 & 0.937 & 5.01 & 4.39 & 2.59 & 1.00 & 3.29 & 2.19 & 3.63 \\
\hline 8877.390 & 0.085 & 5.90 & 4.92 & 1.75 & 1.30 & 3.64 & 2.19 & 3.64 \\
\hline 8878.379 & 0.232 & 5.69 & 5.00 & 1.90 & 1.47 & 3.91 & 1.27 & 4.10 \\
\hline 8879.390 & 0.383 & 5.86 & 5.02 & 1.93 & 0.97 & 3.87 & 1.64 & 3.17 \\
\hline 8880.359 & 0.527 & 5.47 & 4.67 & 0.81 & 1.18 & 2.53 & 1.86 & 2.85 \\
\hline 8881.383 & 0.679 & 4.75 & 4.17 & 1.83 & 1.14 & 3.04 & 1.66 & 3.42 \\
\hline 8882.385 & 0.828 & 5.03 & 4.52 & 1.51 & 1.40 & 3.32 & 1.69 & 2.89 \\
\hline
\end{tabular}


this implies higher levels of global spot coverage than in the previous year.

The phase of light minimum, $\varphi_{\min } \sim 0.02$, is the same as in the previous season, perhaps supporting the hypothesis that the dominant group of spots occurs at the same longitude. The maximum in 1991, however, was double peaked, with a secondary minimum at $\varphi \sim 0.5$. This is no longer seen in 1992.

Interestingly, the colour curves all show negligibly different mean values from the previous epoch, illustrating the small overall effect of the relatively dark starspots on the global colour of the star.

\subsection{Optical flaring}

The large optical flare of 5 September is among the largest yet observed on II Peg. It may be compared in total optical energy to the large flares observed by Doyle et al. (1992a, 1993) whose observed total $U$-band energy were both $\sim 1.810^{35} \mathrm{erg}$, almost identical to the energy detected in the present flare. All of these numbers are lower limits, however, because the light curves were incomplete. The flare light curves were complex and the events themselves long-lived. The flare reported above showed at least three separate light maxima. Its rise lasted $\sim 30 \mathrm{~min}$ and its total duration was $\geq 6.5 \mathrm{hr}$. These parameters are comparable to those of the Doyle et al. flares. Note that this flare was also detected in our UV spectra (Sect. 4.7.1).

\section{3. $H \alpha$}

A comparison of the overall mean $\mathrm{H} \alpha$ line profile from 1992 with that from 1991 (Paper I) is made in Fig. 4 and shows dramatically that there is negligible difference between the two, in spite of the intervening year. This reinforces the conclusion of Paper I that the mean $\mathrm{H} \alpha$ profile of this and, presumably, similar objects, are truly representative of a mean chromosphere.

On the other hand, the results of the EW measurements from the low-resolution data indicate quite clearly that the $\mathrm{H} \alpha$ emission is almost continuously variable at the $50 \%$ level about the mean. This agrees with the data presented in Paper I, where the measured $\mathrm{H} \alpha$ EW's varied between $-0.6 \AA$ and $-1.1 \AA$. Our present data, by comparison, indicates variations over a slightly larger range, i.e. $-0.3 \AA$ to $-1.2 \AA$. Previous authors (cf. Paper I and refs. therein) found values ranging between $-0.2 \AA$ and $-2.0 \AA$. Note that our largest value of $\mathrm{EW}(\mathrm{H} \alpha)$ occurs in a single point a factor of $\sim 2$ larger than the mean, which we consider likely to be due to a flare and is marked as such in Fig. 7. A number of other measurements are marked likewise if they show a large deviation from the local trend. It seems unlikely that the overall slow variations are a result of flaring. The source of these probably lies in gradual changes in the brightness of individual active regions. Previously recorded large EW's were derived from single, isolated spectra and so may also be due to individual flares.

\section{4. $H \beta$}

We have also compared the mean $1992 \mathrm{H} \beta$ profile with that from 1991 in Fig. 5. It is clear that, unlike the same comparison for the $\mathrm{H} \alpha$ spectra, the agreement between the two epoch's profiles is not nearly as good. This is in spite of a good agreement between the nearby photospheric features.

There appears to be an asymmetry towards the blue, in the sense that there appears to be more emission (less absorption) to the blue side of line centre. This same asymmetry was noted in 1991 but at no part of the line was it in net emission above the local continuum at either epoch. Overall $\mathrm{H} \beta$ is more "filled-in" in the current epoch than in 1991.

\section{5. $\mathrm{HeI} \mathrm{D}_{3}$}

In the same way we compare the mean $1992 \mathrm{HeI} \mathrm{D}_{3}$ with that recorded in 1991 from Paper I in Fig. 6. It is immediately obvious that the agreement between the two epochs is poor, with the 1991 mean HeI profile being in strong net absorption and that from 1992 in clear emission. Note that the sense of this difference is similar to that of the $\mathrm{H} \beta$ profiles.

\subsection{Higher Balmer lines and CaII H\&K}

The mean profiles for the CaII H\&K resonance doublet and for the spectral region near $\mathrm{H} \gamma$ and $\mathrm{H} \delta$ lines are given in Fig. 8. Also included in Fig. 8 are spectra taken in 1991 (Paper I) in the same spectral regions. The agreement between the two epochs is excellent with no difference immediately apparent.

These comparisons show a clear trend, i.e. the higher the excitation of the line the greater the relative variability of the line. We reached a similar conclusion in Paper I but based on less comprehensive data.

\subsection{Ultraviolet observations}

Before comparing our current UV line fluxes with those measured at previous epochs, we need to isolate individual flare spectra and omit them from a calculation of the mean flux.

\subsubsection{Flares in UV spectra}

The CIV $\lambda 1548 / 51 \AA$ resonance doublet is the strongest feature in the SWP spectrum of all active late-type stars (Byrne 1995). It is also a sensitive indicator of flares (Doyle et al. 1989b). Examination of Table 9 shows that there are four CIV entries which deviate significantly from the mean. These are indicated in the table as boldface script. 
Similarly in Table 10 one spectrum also stands out and is similarly indicated in the table. Note that the first two SWP and the LWP flare spectra coincide in time with the large optical flare on 5 September (Sect. 4.2). The peak flux $\left(37.510^{-13} \mathrm{erg} \mathrm{cm}^{-2} \mathrm{~s}^{-1}\right.$ averaged over $\left.20 \mathrm{~min}\right)$ is higher than that recorded by Doyle et al. (1992a) for their largest II Peg flare by $\sim 30 \%$.

\subsubsection{Mean UV line fluxes}

The overall mean CIV flux at Earth, excluding the above flares, is $4.8 \pm 0.910^{-13} \mathrm{erg} \mathrm{cm}^{-2} \mathrm{~s}^{-1}$. This may be compared to some previous values. In 1989 Doyle et al. (1992a) recorded a value $6.7 \pm 1.010^{-13} \mathrm{erg} \mathrm{cm}^{-2} \mathrm{~s}^{-1}$, while in 1986, Doyle et al. (1989) found a value $6.2 \pm$ $1.010^{-13} \mathrm{erg} \mathrm{cm}^{-2} \mathrm{~s}^{-1}$. Therefore the 1992 mean flux is significantly lower than either 1986 or 1989 .

\subsection{Microwave observations}

The microwave radiation, which is coronal in origin, shows the largest relative variability of any of the data, apart from the obvious flare. As can be seen in Fig. 9, this variability takes place on all time-scales sampled, i.e. hours to days.

There is a large flare on 13 September (Fig. 9) which reached a peak flux of $\sim 15 \mathrm{mJy}$. Unfortunately the observations terminated while the flare was still in progress. Nevertheless we can place a lower limit to its duration of $4.7 \mathrm{hr}$. Again unfortunately no simultaneous observations at other wavelengths were being made during this time. It may be compared to the peak flux at the same frequency observed by Doyle et al. (1992a,b), i.e. $\sim 8 \mathrm{mJy}$.

The mean "quiescent" flux over the entire observing interval, omitting the flare, is $2.6 \pm 1.5 \mathrm{mJy}$. This may be compared to an upper limit of $\sim 5 \mathrm{mJy}$ from Mutel et al. (1985). Doyle et al. (1992a) observed II Peg over $6 \mathrm{hr}$ on two consecutive nights. On the first night they recorded a secular increase in $5 \mathrm{GHz}$ flux from $\sim 1 \mathrm{mJy}$ to $\sim 2.5 \mathrm{mJy}$ with a mean of $1.92 \pm 0.04 \mathrm{mJy}$. On their second $6 \mathrm{hr}$ night they saw an opposite behaviour, i.e. a decline in $5 \mathrm{GHz}$ flux from $\sim 3.5 \mathrm{mJy}$ to $\sim 2.0 \mathrm{mJy}$ with a mean of $2.34 \pm 0.08 \mathrm{mJy}$. Assuming this is typical of II Peg it is consistent with our current data.

\section{Conclusions}

We have presented one of the most comprehensive multiwavelength data sets on an active late-type star, both in the breadth of its wavelength coverage and its extent in time. It has resulted in a characterisation of mean conditions in the outer atmosphere of II Peg from the photosphere to the corona for comparison with models and with similar data from previous epochs.

We have detected flares on II Peg in optical broadband photometry, in ultraviolet and optical spectroscopy and in microwaves. One flare, recorded in optical broad band photometry and in ultraviolet spectroscopy, is one of the most energetic observed to date on the star.

We reinforce previous evidence that II Peg is highly variable in virtually all spectral signatures, with the degree of variability increasing to higher excitation.

Acknowledgements. Research at Armagh Observatory is supported by a Grant-in-Aid from the Department of Education of Northern Ireland. This research utilised software and hardware provided by the UK SERC STARLINK computing network. MTE, PJA and ACL acknowledge financial support from Armagh Observatory. MTE, PJA and LMS acknowledge support from the European Economic Community's COMETT programme.

\section{References}

Andrews A.D., Rodonó M., Linsky J.L., et al., 1988, A\&A 204, 177

Baars J.W.M., Genzel R., Pauliny-Toth I.I.K., Witzen A., 1977, A\&A 61, 99

Bessell M.S., 1979, PASP 91, 589

Boggess A., et al., 1978, Nat 275, 372

Bromage G.E., 1984, Proc. 4th European IUE Conf., ESA SP218 , p. 473

Byrne P.B., 1983, in "Activity in Red-Dwarf Stars", Byrne P.B. \& Rodonò M. (eds.). Kluwer, p. 157

Byrne P.B., 1986, IBVS, No. 2951

Byrne P.B., 1992a, in "Surface Inhomogeneities on Late-type Stars", Byrne P.B. \& Mullan D.J. (eds.). Springer, p. 3

Byrne P.B., 1992b, in "The Theory of Sunspots", Thomas J.H. \& Weiss N.O. (eds.). Kluwer, Dordrecht, p. 63

Byrne P.B., 1995, in "Flares and Flashes", Proc. IAU Coll. 153, Greiner J., Deurbeck H.W., Gershberg R.E. (eds.). Springer, p. 14

Byrne P.B., Marang F., 1987, Irish AJ 18, 84

Byrne P.B., Doyle J.G., Brown A., Linsky J.L., Rodonó M., 1987, A\&A 176, 284

Byrne P.B., Panagi P.M., Doyle J.G., et al., 1989, A\&A 214, 238

Byrne P.B., Lanzafame A.C., Sarro L.M., Ryans R., 1994, MNRAS 270, 427

Byrne P.B., Panagi P.M., Lanzafame A.C., et al., 1995, A\&A 299, 115 (Paper I)

Cousins A.W.J., 1976, MemRAS 81, 25

Doyle J.G., Butler C.J., Byrne P.B., et al., 1989a, A\&A 223, 219

Doyle J.G., Byrne P.B., van den Oord G.H.J., 1989b, A\&A 224,153

Doyle J.G., Kellett B.J., Byrne P.B., et al., 1991, MNRAS 248, 503

Doyle J.G., Kellett B.J., Butler C.J., et al., 1992a, A\&AS 96, 351

Doyle J.G., van den Oord G.H.J., Kellett B.J., 1992b, A\&A 262,533

Doyle J.G., Mathioudakis M., Murphy H.M., Avgoloupis S., Mavridis L.N., Seiradakis J.H., 1993, A\&A 278, 499

Elliott K.H., 1996, MNRAS 281, 158

Gershberg R.E., 1972, ApSS 19, 75

Giddings J.R., 1983, STARLINK User Note No. 37, Rutherford Appleton Labs. 
Glass I.S., 1985, Irish AJ 17, 1

Howarth I.D., Murray M.J., 1987, STARLINK User Note No. 50, Rutherford Appleton Labs.

IDL Users' Guide, 1985, Research Systems Inc. Boulder

Johnson 1966, A\&AR 4, 193

Mavridis L.N., Asteriadis G., Mahmoud F.M., 1982, Compendium in Astron., Mariolopoulos E.G., Theocaris P.S., Mavridis L.N. (eds.). Reidel, Dordrecht, p. 253

Menzies J.W., Marang F., Laing J.D., Coulson I.M., Englebrecht C.A., 1991, MNRAS 248, 642

Meyerdicks H., 1993, STARLINK User Note No.86.9, Rutherford Appleton Labs.

Mutel R.L., Lestrade J.F., Preston R.A., Phillips R.B., 1985, ApJ 289, 262

Padin S., Davis R.J., Lasenby A.N., 1987, MNRAS 224, 685

Rodonó M., Cutispoto G., Pazzani V., et al., 1986, A\&A 165, 135

Rodonó M., Byrne P.B., Neff J.E., et al., 1987, A\&A 176, 284

Strassmeier K.S., Hall D.S., Fekel F.C., Scheck M., 1993, A\&AS 100, 173

Tody D., et al., 1986, IRAF Users' Manual, National Optical Astronomical Observatories

Vogt S.S., 1981, ApJ 247, 975
E-mail addresses:

PBB: pbb@star.arm.ac.uk

LMS: lms@laeff.esa.es

MTE: mte@star.arm.ac.uk

PJA: pja@star.arm.ac.uk

JGD: jgd@star.arm.ac.uk

HMM: hmm@star.arm.ac.uk

ACL: acl@sunct.ct.astro.it

HAA: abdul@usm.my

RES: res@jb.man.ac.uk

JEN: jneff@astro.sunysb.edu

JHS: jhs@astro.auth.gr

SA: avgoloup@astro.auth.gr

RDJ: rdj@astro.keele.ac.uk

KHE: khe@star.sr.bham.ac.uk

MJA: mam@iac.es

CL: clh@iac.es 